\title{
SHORT- AND LONG-RUN LINKAGES BETWEEN EMPLOYMENT GROWTH, INFLATION AND OUTPUT GROWTH: EVIDENCE FROM A LARGE PANEL
}

\author{
Marinko ŠKAREa, Guglielmo Maria CAPORALE ${ }^{\mathrm{b}}$ \\ ${ }^{a}$ Faculty of economics and tourism "Dr. Mijo Mirkovic", Juraj Dobrila University of Pula, Pula, Croatia \\ ${ }^{\mathrm{b}}$ Centre for Empirical Finance, Brunel University, West London UB8 3PH, United Kingdom
}

Received 06 October 2013; accepted 16 March 2014

\begin{abstract}
This study examines the short- and long-run linkages between employment growth, inflation and output growth applying panel cointegration and causality tests to data for 119 countries over the period 1970-2010. We find evidence of positive Granger causality running from output growth to employment growth in the short run. Employment growth Granger causes output growth with a negative sign in the long run. Inflation Granger causes employment and output growth positively in the short run and negatively in the long run.
\end{abstract}

Keywords: employment, inflation, output, Granger causality.

JEL Classification: C23, E24, E31, E60.

\section{Introduction}

The seminal study by Phillips (1962) stressed the importance for policy-makers of understanding the joint dynamics of employment growth, inflation and output growth in order to improve macroeconomic performance. Whilst numerous papers analyse these variables individually (see, for example, Phillips (1958), Tobin (1997), Targetti (1992), Okun (1981, 1980), Phelps and Zoega (1998), Friedman (1968), Friedman and Schwartz (1971), only a few examine their simultaneous interactions (Scott, McKean 1964), Phelps (1967), Gordon et al. (1977), Gordon (1991), Phelps and Zoega (1998), Nickell (1998), Acemoglu and Scott (1994)). As Phillips pointed out (1962), the fact that they are both targets and policy instruments makes knowledge of their relationships even more crucial in order to avoid "overshooting" or "undershooting" of the targeted "equilibrium" values.

Corresponding author Marinko Skare

E-mail:mskare@unipu.hr 
This paper aims to provide new empirical evidence on the linkages between these variables by applying panel VECM techniques and carrying out causality tests in the context of an extensive balanced panel of annual data on prices, employment, and output (in annual percentage changes) from 1970 to 2010 for 119 countries. This is in contrast to earlier papers typically estimating VARs for individual countries or analysing small panels of OECD or EU countries (see Hu 2004; Marelli, Signorelli 2010; Loboguerrero, Panizza 2006; Sill 2011; Lucas, Rapping 1969; Hossain 2005; Arato 2009; Motley 1998). An exception is a previous study by Caporale and Škare (2011) exploring the joint behaviour of employment growth, inflation and output growth in a panel cointegration framework. The present paper expands their analysis by estimating VECM specifications at both panel and individual country level, thereby providing both aggregate and disaggregate evidence; in addition, it also tests for both short- and longrun causality. As in Scott and McKean (1964), it considers a three-variable model, unlike the majority of existing studies that focus instead on the linkages between only two of the variables in question (see Barro 1995; Ewing 1999; Michie, Smith 1997). To preview our results, we find clear evidence of both short- and long-run linkages between the three variables analysed.

The structure of the paper is as follows. Section 1 provides a brief literature review. Section 2 describes the econometric framework and estimation. Section 3 focuses on the Granger causality test results. Section 4 offers some concluding remarks.

\section{Literature review}

In his famous studies on the UK economy Phillips $(1958,1962)$ highlighted the importance of analysing the relationships between unemployment, the rate of change of money wage rates and output growth as well. The validity of the unemployment-inflation relationship he detected and its implications for monetary policy were then questioned by Phelps (1967), Phelps and Zoega (1998), Friedman (1968) and Friedman and Schwartz (1971).

Among subsequent studies, Okun (1981) focused on the inflation-unemployment tradeoff faced by policy-makers (see also Tobin 1997, 1987, 1982), whilst Kaldor in Targetti (1992) modelled unemployment as a function of the marginal labour productivity and the associated labour costs (a decrease in marginal labour productivity or an increase in labour costs result in higher unemployment).

Scott and McKean (1964) saw employment and price stability as alternative policy goals (1964). According to Landmann (2004), employment and productivity are strongly and positively correlated over the business cycle in a pro-cyclical way. Marelli and Signorelli (2010) found evidence that high employment growth leads to slower productivity growth in the EU area. Pissarides and Vallanti (2004) reported a positive relationship between TFP growth and employment. Phelps (1994) and Ball and Moffitt (2001) argued that growth has only temporary, short-run effects on employment. Oil shock as found in Çatik and Karaçuka (2012) exert strong influence on inflation dynamics. Inflationary pressures appears to be closely connected with gross average wage dynamics, debt accumulation and consumption growth (Bratu 2012). Linearity and constancy assumption in the augmented Phillips curve model in relation to expected inflation should also be accounted (Šergo et al. 2012). The impact of oil shock on prices is studied in Semko (2013) with money - income link examined in Bozoklu (2013) for Turkey. 
Barro (2013) presented evidence of the adverse effects of inflation on growth, while Andres and Hernando (1999) found a negative correlation between the two. Bruno and Easterly (1996) found a short- to medium-term negative relationship between inflation and growth but no evidence that discrete inflation jumps harm growth in the long run. Hooker (2002) reported evidence of a backward-looking Phillips' curve for the US, a finding confirmed by Nakov and Pescatori (2010). Only a recent paper by Caporale and Škare (2011) studies the simultaneous relationships between employment growth, inflation and GDP growth in a panel cointegration framework. Similar research exploring scientifically acceptable law of dynamics between output, employment and inflation can be found in Škare (2010).

\section{Econometric analysis}

We use a balanced panel of annual data on prices, employment, and output (in annual percentage changes) from 1970 to 2010 for 119 countries $^{1}$. These are taken from the USDA International macroeconomic dataset (historical data files) and the Conference board total economy database 2011.

\section{A. Testing for integration (nonstationarity)}

As a first step, we check the order of integration of the series using the panel unit root tests developed by Im et al. (2003), Pesaran (2004, 2007), Pesaran et al. (1999), Pesaran and Smith (1995), Maddala and Wu (1999), Hadri (2000).

Im, Pesaran and Shin (IPS) allow for heterogeneity in $\rho_{i}$ by adopting the following specification (see also Hurlin, Mignon 2007):

$$
\Delta y_{i, t}=\alpha_{i}+\rho_{i} y_{i, y-1}+\sum_{z=1}^{p_{i}} \beta_{i, z} \Delta y_{i, t-z}+\varepsilon_{i, t} .
$$

Maddala, Wu (1999) and Choi (2001) propose a Fisher-type test based on the p-values from the individual unit root tests taking the form (MW):

$$
\begin{gathered}
-2 \sum_{i=1}^{N}\left(\ln p_{i}\right) \sim \chi^{2}(2 N) ; \\
P_{m}=\frac{1}{2 \sqrt{N}} \sum_{i=1}^{N}\left(-2 \ln p_{i}-2\right) .
\end{gathered}
$$

\footnotetext{
1 The countries included in the panel are the following: Albania, Algeria, Angola, Argentina, Armenia, Australia, Austria, Azerbaijan, Bahrain, Bangladesh, Barbados, Belarus, Belgium, Bosna \& Hercegovina, Brazil, Bulgaria, Burkina Faso, Cambodia, Cameron, Canada, Central African Republic, Chile, China, Colombia, Congo, Democratic Republic of Costa Rica, Cote d'Ivore, Croatia, Cyprus, Czech Republic, Denmark, Ecuador, Egypt, Estonia, Ethiopia, Finland, France, Georgia, Germany, Ghana, Greece, Guatemala, Hungary, Iceland, India, Indonesia, Iran, Iraq, Ireland, Israel, Italy, Jamaica, Japan, Jordan, Kazakhstan, Kenya, Korea South, Kuwait, Kyrgyzstan, Latvia, Lithuania, Luxembourg, Macedonia, Madagascar, Malawi, Malaysia, Mali, Malta, Mexico, Moldova, Morocco, Mozambique, Myanmar, The Netherlands, New Zealand, Niger, Nigeria, Norway, Oman, Pakistan, Peru, The Philippines, Poland, Portugal, Romania, Russia, St.Lucia, Saudi Arabia, Senegal, Serbia, Singapore, Slovakia, Slovenia, South Africa, Spain, Sri Lanka, Sudan, Sweden, Switzerland, Syria, Taiwan, Tajikistan, Tanzania, Thailand, Trinidad \& Tobago, Tunisia, Turkey, Turkmenistan, Uganda, Ukraine, United Arab Emirates, United Kingdom, USA, Uruguay, Uzbekistan, Venezuela, Vietnam, Yemen, Zambia.
} 
Hadri (2000) suggests a different panel unit root test based on the null of stationarity allowing for individual specific variances and correlation patterns. His $(\mathrm{H})$ test takes the form:

$$
y_{i t}=\delta_{m i} d_{m t}+\varepsilon_{i t} .
$$

We use Baum's (2001) version of Hadri's test (2000), which is a residual-based Langrange multiplier (LM) test for a unit root in panel data under the null that the observed series are stationary around a deterministic level or a deterministic trend.

The result of the IPS, MW unit root tests are presented in Table 1.

Table 1. Panel unit root tests

\begin{tabular}{ccc}
\hline \multirow{2}{*}{ Variables } & \multicolumn{2}{c}{ Levels } \\
\cline { 2 - 3 } & IPS & MW \\
\hline$y$ & $-25.87^{\star *}$ & $2182^{\star *}$ \\
$p$ & $-17.02^{\star *}$ & $1752^{\star *}$ \\
$e$ & $-19.96^{\star *}$ & $1248^{\star *}$ \\
\hline
\end{tabular}

Notes: Variables in levels, ${ }^{*}, * *$ indicate $5 \%, 1 \%$ rejection levels. IPS, MW tests for a unit root. $\mathrm{H}$ test of the stationarity null.

IPS and MW (see Table 1) reject the unit root null, whilst the $\mathrm{H}$ test (see Table 2) strongly rejects the null of stationarity in the panel for the series in levels but fails to reject for the differenced series.

Table 2. Hadri unit root test

\begin{tabular}{ccccccc}
\hline \multirow{2}{*}{ Variables } & \multicolumn{3}{c}{ Levels } & \multicolumn{3}{c}{ First differences } \\
\cline { 2 - 6 } & $(1)$ & $(2)$ & $(3)$ & \multicolumn{1}{c}{$(1)$} & $(2)$ & $(3)$ \\
\hline$y$ & $26.551^{\star * *}$ & $19.476^{\star * *}$ & $14.650^{\star * *}$ & -12.140 & -11.655 & 20.638 \\
$p$ & $20.378^{* * *}$ & $42.813^{\star * *}$ & $15.095^{\star * *}$ & -13.102 & -8.712 & 22.979 \\
$e$ & $14.703^{* * *}$ & $38.319^{* * *}$ & $15.526^{* * *}$ & 14.703 & 38.319 & 15.526 \\
\hline
\end{tabular}

Notes: ${ }^{*},{ }^{* *}$ indicate $5 \%, 1 \%$ rejection levels. Hadri test under the null of stationarity in the model. (1) denotes the homoscedasticity assumption, (2) denotes the heteroscedasticity assumption, (3) denotes the serial correlation assumption.

The ambiguity of the unit root test results arises from the interdependence between the series in the panel. As shown by O'Connell (1998), panel unit root tests over-reject the unit root null in the presence of cross-unit cointegration displaying "false" high power. Breuer et al. $(2001,2002)$ discuss the power of panel unit root test in such a case, pointing out that they have lower power when testing the unit root null as opposed to that of stationarity. Persyn and Westerlund (2008) and Westerlund (2008) show that the empirical failure to reject the unit root null does not definitely establish its presence. Among recent studies, Tatoglu (2011) finds a unit root in both unemployment and GDP for a panel of EU countries; using non-parametric tests, Holl and Kunst (2011) also find evidence of a unit root in unemployment; Narayan, P., Narayan, S. (2010) report the presence of a unit root in inflation in $17 \mathrm{EU}$ countries. Barbieri (2008) recommends to use both the IPS and MW tests for the null of a unit root as well as tests for the null of stationarity $(\mathrm{H})$ in the case of a heterogeneous panel. Hadri 
and Rao $(2008,2009)$ using their stationarity test (2008) find that the null of stationarity with a break cannot be rejected for 4 out of 14 variables $^{2}$. Hurlin (2004) found that the unit root hypothesis cannot be rejected when taking into account cross-sectional dependence for most variables including GDP, wages, real wages, the unemployment rate and the money stock.

In our case, since the $\mathrm{H}$ test strongly rejects at the $1 \%$ level the null of stationarity for the levels and fails to reject it for the first differences under the assumptions of homoscedasticity, heteroscedasticy and serial dependence respectively in the disturbances, we conclude that series in the panel are integrated of order I(1), and then proceed to test for possible long-run relationships using panel cointegration tests.

\section{B. Panel cointegration tests and FMOLS, DOLS estimation}

We carry out the Nyblom-Harvey, Fisher-Johansen, Pedroni, Westerlund and Kao cointegration tests. Nyblom and Harvey (2000) test for common stochastic trends in the panel under the null of zero common trends as a proxy for cointegration relationship. Their test takes the form:

$$
\zeta_{k, n}=\lambda_{k+1}+\ldots+\lambda_{n}
$$

The Fisher-based Johansen panel cointegration test $(1995,1988)$ developed by Maddala and $\mathrm{Wu}(1999)$ uses individual cross-section test results as follows:

$$
\Delta Y_{i, t}=\prod_{i} y_{i, t-1}+\sum_{k=1}^{n} T_{k} \Delta Y_{i, t-k}+u_{i, t}
$$

Pedroni's $(1999,2004)$ tests for cointegration are based on the estimated residual in the form:

$$
e_{i t}=\rho_{i} e_{i t-1}+\sum_{j=1}^{p_{i}} \psi_{i j} \Delta e_{i t-1}+v_{i t} .
$$

Kao (1999) develops a similar residual-based panel cointegration test under the null of stationarity of the residuals with homogenous variance of the innovation process $\varepsilon_{i t}$ :

$$
\hat{\varepsilon}_{i t}=\rho \hat{\varepsilon}_{i t-1}+\sum_{j=1}^{p} v_{j} \Delta \hat{\varepsilon}_{i t-j}+v_{i t} .
$$

Westerlund (2008) uses an error-correction panel cointegration test for error correction in individual panel member and in the full panel:

$$
\Delta y_{i t}=\delta_{i}^{\prime} d_{t}+\alpha_{i} y_{i t-1}+\lambda_{i}^{\prime} x_{i t-1}+\sum_{j=1}^{p_{i}} \alpha_{i j} \Delta y_{i t-j}+\sum_{j=0}^{p_{i}} \gamma_{i j} \Delta x_{i t-j}+e_{i t} .
$$

\footnotetext{
2 Real GDP, Nominal GDP, Real per capita GDP, Industrial production, Employment, Unemployment rate, GDP de-
} flator, Consumer prices, Wages, Real wages, Money stock, Velocity, Bond yield, Common stock prices. 
The panel cointegration test results are presented in Table 3, with the lag length selected on the basis of the Akaike information criterion with individual intercepts and trend.

Table 3. Panel cointegration tests results

\begin{tabular}{cccccccccc}
\hline & \multicolumn{2}{c}{ Nyblom-Harvey } & \multicolumn{2}{c}{ Fisher-Johansen } & \multicolumn{2}{c}{ Pedroni } & \multicolumn{2}{c}{ Westerlund } & Kao \\
\cline { 2 - 10 } & $\mathrm{F}$ & $\mathrm{T}$ & Trace & Max & $\mathrm{F}$ & $\mathrm{T}$ & $\mathrm{F}$ & $\mathrm{T}$ & $\mathrm{F}$ \\
\hline$y$ & $7.48^{* *}$ & $5.96^{* *}$ & $1589^{* *}$ & $1175^{* *}$ & $-30.91^{* *}$ & $-35.03^{* *}$ & $-18.78^{* *}$ & $-24.26^{* *}$ & $-13.77^{* *}$ \\
$p$ & $7.48^{* *}$ & $5.96^{* *}$ & $1317^{* *}$ & $1214^{* *}$ & $-17.45^{* *}$ & $-18.36^{* *}$ & $-11.41^{* *}$ & $-20.15^{* *}$ & $-18.92^{* *}$ \\
$e$ & $6.82^{* *}$ & $5.97^{* *}$ & $1130^{* *}$ & $954^{* *}$ & $-39.83^{* *}$ & $-48.44^{* *}$ & $-13.28^{* *}$ & $-19.27^{* *}$ & $-12.29^{* *}$ \\
\hline
\end{tabular}

Notes: Variables in levels, ${ }^{*},{ }^{* *}$ indicate $5 \%, 1 \%$ rejection levels.

It can be seen that all panel cointegration tests strongly reject the null of no cointegration in favour of a long-run relationship between employment growth, inflation and output growth. This holds for both the individual panel units and the full panel.

Following Pedroni (2001), we also apply group-mean panel FMOLS and DOLS methods. The FMOLS estimator takes the form:

$$
\hat{\beta}_{G F M}^{\star}=N^{-1} \sum_{i=1}^{N}\left(\sum_{t=1}^{T}\left(p_{i t}-\bar{p}_{i}\right)^{2}\right)^{-1} \times\left(\sum_{t=1}^{T}\left(p_{i t}-\bar{p}_{i}\right) s_{i t}^{\star}-T \hat{\gamma}_{i}\right),
$$

and the DOLS one

$$
\hat{\beta}_{G D}^{*}=\left[N^{-1} \sum_{i=1}^{N}\left(\sum_{t=1}^{T} z_{i t} z_{i t}^{\prime}\right)^{-1}\left(\sum_{t=1}^{T} z_{i t} \tilde{s}_{i t}\right)^{-1}\right]_{1} .
$$

The results overwhelmingly reject the null of no cointegration (see Table 4) in favour of a long-run relationship, with only a few exceptions (see Table A1).

Table 4. FMOLS and DOLS group-mean panel test results

\begin{tabular}{lcccc}
\hline \multicolumn{1}{c}{ Panel results } & FMOLS & t-stat & DOLS & t-stat \\
\hline $\begin{array}{l}\text { Without time dummies } \\
\text { Between }\end{array}$ & 0.03 & $-1589.83^{\star *}$ & 0.03 & $-1368.77^{\star *}$ \\
\hline $\begin{array}{l}\text { With time dummies } \\
\text { Between }\end{array}$ & -0.01 & $-2722.07^{\star *}$ & -0.01 & $-2141.17^{\star *}$ \\
\hline
\end{tabular}

Notes: Variables in levels, ${ }^{*}, * *$ indicate $5 \%, 1 \%$ rejection levels.

\section{Model specification}

We aim to analyse the relationship between employment growth, inflation and output growth in 119 countries over the period 1970-2010, i.e.:

$$
\begin{gathered}
y_{i t}=a_{i}^{\prime}+a_{t}^{\prime}+\hat{a}_{1 i} x_{1 i, t}+\hat{a}_{2 i} x_{2 i, t}+\ldots+\hat{a}_{M i} x_{M i, t}+\varepsilon_{i t}, \\
\text { for } t=1, \ldots, T ; \quad i=1, \ldots, N ; \quad m=1, \ldots, M,
\end{gathered}
$$

where $y_{i t}$ is the annual growth rate of real output in country $i$ and year $t, p_{i t}$ is the annual change of the price level, $e_{i t}$ the annual change in the employment level and $u_{i t}$ the error term. 
Following Pedroni $(2004,1999)$, we estimate a panel VECM model:

$$
\Delta y_{i t}=\theta_{1 i}+\lambda_{1 i} E C_{i, t-1}+\sum_{k=1}^{m} \theta_{11 i k} \Delta y_{i, t-k}+\sum_{k=1}^{m} \theta_{12 i k} \Delta p_{i, t-k}+\sum_{k=1}^{m} \theta_{13 i k} \Delta e_{i, t-k}+u_{1 i t}
$$

and then test for multivariate causality with lag length $\mathrm{m}(\mathrm{SIC}=2)$ to examine multivariate causality within the VECM framework using Wald tests (F test) of the null $H_{0}: \theta_{12 i k}, \theta_{13 i k}=0, H_{0}: \theta_{22 i k}, \theta_{23 i k}=0, H_{0}: \theta_{31 i k}, \theta_{32 i k}=0$ and also of $H_{0}: \lambda_{1 i}, \lambda_{2 i}, \lambda_{3 i}=0$ for all $i$ and $k$ in (22). Table 5 and A2 display the results of the multivariate Granger causality analysis.

\section{Granger causality tests}

Table 5 summarises the results of short- and long-run Granger causality tests.

Table 5. Summary of the findings from the Panel Granger Causality Analysis

\begin{tabular}{|c|c|c|c|}
\hline \multirow{2}{*}{$\begin{array}{l}\text { Dependent } \\
\text { variable }\end{array}$} & \multirow{2}{*}{$\begin{array}{l}\text { Independent } \\
\text { variable }\end{array}$} & \multicolumn{2}{|c|}{ Granger causality } \\
\hline & & Short run & Long run \\
\hline$y$ & $e$ & $\begin{array}{l}\text { Employment has limited impact on } \\
\text { growth in the short run. Increasing } \\
\text { employment slowly boosts growth. } \\
\text { In the short run there is strong and } \\
\text { significant positive Granger causal- } \\
\text { ity running from growth to employ- } \\
\text { ment. The impact of employment } \\
\text { on growth is statistically significant } \\
\text { only for very few countries. }\end{array}$ & $\begin{array}{l}\text { There is clear evidence of long- } \\
\text { run causality running from em- } \\
\text { ployment to output growth in the } \\
\text { long run with a negative sign. For } \\
\text { most of the countries in the panel } \\
\text { Granger causality is unidirectional } \\
\text { running from employment growth } \\
\text { to output growth. }\end{array}$ \\
\hline$p$ & $e$ & $\begin{array}{l}\text { Inflation positively Granger causes } \\
\text { employment in the short run. This } \\
\text { confirms the idea that inflation has } \\
\text { a beneficial impact on employment } \\
\text { in the short run. Increasing employ- } \\
\text { ment in turn has a positive effect } \\
\text { on output growth in the short run. }\end{array}$ & $\begin{array}{l}\text { We find negative Granger causality } \\
\text { running from inflation to employ- } \\
\text { ment in the long run, which can be } \\
\text { rationalised by arguing that, as in- } \\
\text { flation uncertainty rises, agents be- } \\
\text { come more risk averse. In } 8 \text { coun- } \\
\text { tries causality is bidirectional. }\end{array}$ \\
\hline$y$ & $p$ & $\begin{array}{l}\text { For } 38 \text { countries we find evidence } \\
\text { that inflation Granger causes out- } \\
\text { put growth in the short run and } \\
\text { therefore price increases have a } \\
\text { positive effect on output growth } \\
\text { in the short run. }\end{array}$ & $\begin{array}{l}\text { Price increases in the long run } \\
\text { have a negative effect on growth. } \\
\text { We find unidirectional causality } \\
\text { running from inflation to growth } \\
\text { in } 81 \text { countries and bidirectional } \\
\text { one in } 14 \text {. }\end{array}$ \\
\hline
\end{tabular}

Source: Own calculations.

We test for the presence of both short- and long-run causality as well as for the presence of both (joint Granger causality). To test for the latter we carry out $\mathrm{F}$ tests for the joint significance of the lags of the explanatory variables (short-run) and the lagged error correction term (long-run equilibrium) ${ }^{3}$.

3 Specifically, we test if changes in $\mathrm{X}$ are a function of past changes in both $\mathrm{X}$ and $\mathrm{Y} H_{0}: \theta_{12 i k}, \theta_{13 i k}=0, H_{0}: \theta_{22 i k}$, $\theta_{23 i k}=0, H_{0}: \theta_{31 i k}, \theta_{32 i k}=0$ and whether there is an adjustment process towards the long-run equilibrium $\left(E C_{i, t-1}\right)$ i.e. $H_{0}: \lambda_{1 i}, \lambda_{2 i}, \lambda_{3 i}=0$. 
The null hypothesis that inflation does not Granger cause employment growth cannot be rejected in the short run. However, in the long run it can be rejected with inflation negatively affecting employment growth (causality being unidirectional in most cases). For small open economies, transition and African countries, negative unidirectional Granger causality from inflation to employment growth is detected in the long run (negative long-run effects). Testing for joint Granger causality (short- and long-run) indicates that inflation positively Granger causes employment growth in most cases (in 47 countries at the 1, 5 and 10\% level). In the short run inflation has a less significant (positive) impact on employment growth, and only in a few countries (the US, the former Soviet republics and other transition countries). Bidirectional Granger causality between inflation and employment is found for 43 countries in the panel.

The null hypothesis that output growth does not Granger cause employment growth in the short run cannot be rejected in most cases. The Granger causality relation between output growth and employment growth is significant but not as strong as the inflation-output growth one. The results differ across the countries in the panel. Output growth Granger causes employment growth in 30 countries only, whilst in the other cases causality runs in the opposite direction. For output and employment growth, the null hypothesis that output growth does not Granger cause employment growth in the short run is not rejected (at the $1,5 \%$ significance level). Unidirectional causality is found in the long run from employment growth to output growth. The joint Granger causality tests provide evidence of positive effects of employment growth on output growth in both the short and the long run.

The null hypothesis that inflation has no short-run effects on (does not Granger cause) output growth is rejected for a large number of countries in the panel. The evidence suggests that inflation is conducive to growth in the short run but damaging in the long run. In fact, the null of no Granger causality between inflation and output growth is rejected overwhelmingly, the results implying a negative long-run relationship. In most countries causality is unidirectional and runs from inflation to output growth. However, in countries such as Greece, Iceland, Iran, Kyrgyzstan, Latvia, Angola, Armenia, Belarus, Lithuania etc., there is evidence of bidirectional joint Granger causality. It is noteworthy that in countries vulnerable to external shocks, such as Greece and Iceland, both long-run and joint Granger causality between inflation and output growth are stronger.

Overall, one can interpret these results as suggesting that positive growth during moderate inflation periods is likely to offset negative growth rate associated with higher inflation periods. It would be worthwhile to determine the threshold at which the inflation rate starts affecting growth negatively.

The Granger causality analysis for the 119 countries in the panel considered individually produces some interesting results (see Table A2). In particular, past values of GDP appear to predict current price movements both in the short and in the long run. The links between inflation and output growth for a large number of countries in the panel appear to be bidirectional. Price changes therefore also influence output growth movements.

On the whole, causality linkages appear to be quite different in the short run as opposed to the long run. Also, the evidence provides broad support to the existence of dynamic relationships in the Phillips' and Okun's tradition. It would be interesting also to investigate (using Markov Switching procedures) how these respond to changes in the economic environment. 


\section{Conclusions}

The aim of this study was to examine the relationship between employment growth, inflation and output growth in the tradition of Phillips $(1958,1962)$ using panel VECM methods and Granger causality tests. The analysis builds on Caporale and Škare (2011), who carry out panel cointegration tests using an extensive panel including 119 countries, in that it estimates individual country models as well and also conducts Granger causality tests (both in the short and long run). The existence of strong linkages is confirmed by our results (especially the FMOLS and DOLS ones), which are informative for policy-makers and offer a number of useful insights. For example, employment growth is found to have a positive impact on output growth in the short run but a negative one in the long run (as output increases at a slower rate than productivity). The link between employment and output growth is clearly positive in the short run, with causality running from the former to the latter in most cases. By contrast, the link becomes negative in the long run.

The inflation-output growth relationship is undoubtedly positive in the short run, i.e. inflation is beneficial to growth. In the long run, instead, price volatility and uncertainty appear to affect output growth adversely. Inflation positively Granger cause employment in the short run, so it can boost employment temporarily. However, in the long run it has damaging effects, leading to lower output and employment growth, and consequently the inflation-employment growth relationship becomes negative. Obviously, changes can occur over time, and one should therefore investigate whether or not that implies that the Phillips curve relationship does not hold any longer; however, this issue is beyond the scope of the present paper and is left for future research.

\section{Acknowledgments}

We thank the editor and two anonymous reviewers for their constructive comments, which helped us to improve the manuscript.

\section{References}

Acemoglu, D.; Scott, A. 1994. Asymmetries in the cyclical behaviour of UK labour markets, The Economic Journal 104(427): 1303-1323. http://dx.doi.org/10.2307/2235450

Andrés, J.; Hernando, I. 1999. Does inflation harm economic growth? Evidence from the OECD, in M. Feldstein (Ed.). The costs and benefits of price stability. University Of Chicago Press, 315-348.

Arato, H. 2009. Long-run relationship between inflation and growth in a New Keynesian framework, Economics Bulletin 29(3): 1-9.

Ball, L. M.; Moffitt, R. A. 2001. Productivity growth and the Phillips curve. National Bureau of Economic Research Cambridge, Mass. 52 p. http://dx.doi.org/10.3386/w8421

Barbieri, L. 2008. Panel cointegration tests: a survey, Rivista Internazionale di Scienze Sociali 116(1): 3-36.

Barro, R. J. 1995. Inflation and economic growth, Bank of England Quarterly Bulletin: 166-176.

Barro, R. J. 2013. Inflation and economic growth, Annals of Economics and Finance 14(1): 121-144. Society for AEF.

Baum, C. F. 2001. HADRILM: Stata module to perform Hadri panel unit root test. Statistical Software Components, Boston College Department of Economics. 
Bozoklu, Ş. 2013. Money, income, and causality: an examination for the Turkish economy, Ekonomska istraživanja - Economic Research 26(1): 171-182.

Bratu, M. 2012. The reduction of uncertainty in making decisions by evaluating the macroeconomic forecast performance in Romania, Ekonomska istraživanja - Economic Research 25(2): 239-262.

Breuer, J. B.; McNown, R.; Wallace, M. 2002. Series specific unit root tests with panel data, Oxford Bulletin of Economics and statistics 64(5): 527-546. http://dx.doi.org/10.1111/1468-0084.00276

Breuer, J. B.; McNown, R.; Wallace, M. 2001. Misleading inferences from panel unit root tests with an illustration from purchasing power parity, Review of International Economics 9(3): 482-493. http://dx.doi.org/10.1111/1467-9396.00294

Bruno, M.; Easterly, W. 1996. Inflation and growth: in search of a stable relationship, Review Federal Reserve Bank of St. Louis 78: 1-8.

Çatik, A. N.; Karaçuka, M. 2012. Oil pass-through to domestic prices in Turkey: does the change in inflation regime matter?, Ekonomska istraživanja - Economic Research 25(2): 277-296.

Caporale, G. M.; Škare, M. 2011. Employment growth, inflation and output growth: was Phillips right? Evidence from a dynamic panel, CESifo Working Paper No. 3502, 1-27.

Choi, I. 2001. Unit root tests for panel data, Journal of International Money and Finance (20): 249-272. http://dx.doi.org/10.1016/S0261-5606(00)00048-6

Ewing, B. T. 1999. Employment and economic performance: jobs, inflation, and growth, Journal of Labor Research 20(4): 622-624. http://dx.doi.org/10.1007/s12122-999-0026-x

Friedman, M. 1968. The role of monetary policy, American economic review 58(1):1-17.

Friedman, M.; Schwartz, A. J. 1971. A monetary history of the United States, 1867-1960. Princeton University Press. 888 p.

Gordon, R. J. 1991. The Phillips curve now and then. National Bureau of Economic Research Cambridge, Mass., USA, 1-20.

Gordon, R. J.; Perry, G.; Modigliani, F.; Okun, A.; Wachter, M.; Kouri, P.; Phelps, E.; Sims, C. 1977. Can the inflation of the 1970s be explained?, Brookings Papers on Economic Activity (1): 253-279. http://dx.doi.org/10.2307/2534262

Hadri, K. 2000. Testing for stationarity in heterogeneous panel data, The Econometrics Journal 3(2):148-161. http://dx.doi.org/10.1111/1368-423X.00043

Hadri, K.; Rao, Y. 2008. Panel stationarity test with structural breaks, Oxford Bulletin of Economics and statistics 70(2): 245-269. http://dx.doi.org/10.1111/j.1468-0084.2008.00502.x

Hadri, K.; Rao, Y. 2009. Are OECD macroeconomic variables trend stationary? Evidence from panel stationarity tests allowing for a structural break and cross sectional dependence, The Singapore Economic Review (SER) 54(03): 427-440. http://dx.doi.org/10.1142/S0217590809003410

Holl, J.; Kunst, R. M. 2011. Unit root in unemployment-new evidence from nonparametric tests, Applied Economics Letters 18.6 (2011): 509-512. http://dx.doi.org/10.1080/13504851003725934

Hooker, M. A. 2002. Are oil shocks inflationary? Asymmetric and nonlinear specifications versus changes in regime, Journal of Money, Credit and Banking 34(2): 540-561. http://dx.doi.org/10.1353/mcb.2002.0041

Hossain, A. 2005. The granger-causality between money growth, inflation, currency devaluation and economic growth in Indonesia: 1954-2002, International Journal of Applied Econometrics and Quantitative Studies 2(3): 45-67.

$\mathrm{Hu}, \mathrm{A}$. 2004. Economic growth and employment growth in China (1978-2001), Asian Economic Papers 3(2): 166-176. http://dx.doi.org/10.1162/1535351044193376

Hurlin, C. 2004. Nelson and Plosser revisited: a re-examination using OECD panel data. Document de Recherche 23 Laboratoire d'Economie d'Orleans, 1-44. 
Hurlin, C.; Mignon, V. 2007. Second generation panel unit root tests. Working Paper. CEPII and EconomiX-CNRS, 1-24.

Im, K. S.; Pesaran, M. H.; Shin, Y. 2003. Testing for unit roots in heterogeneous panels, Journal of Econometrics 115(1): 53-74. http://dx.doi.org/10.1016/S0304-4076(03)00092-7

Johansen, S. 1988. Statistical analysis of cointegration vectors, Journal of economic dynamics and control 12(2-3): 231-254. http://dx.doi.org/10.1016/0165-1889(88)90041-3

Johansen, S. 1995. Likelihood-based inference in cointegrated vector autoregressive models. Oxford University Press, USA. 280 p. http://dx.doi.org/10.1093/0198774508.001.0001

Kao, C. 1999. Spurious regression and residual-based tests for cointegration in panel data, Journal of Econometrics 90(1): 1-44. http://dx.doi.org/10.1016/S0304-4076(98)00023-2

Landmann, O. 2004. Employment, productivity and output growth, Employment Strategy Papers 17: 1-61.

Loboguerrero, A.; Panizza, U. 2006. Does inflation grease the wheels of the labor market?, Contributions in Macroeconomics 6(1): 1-28. http://dx.doi.org/10.2202/1534-6005.1450

Lucas, R. E.; Rapping, L. A. 1969. Real wages, employment, and inflation, The Journal of Political Economy 77(5): 721-754. http://dx.doi.org/10.1086/259559

Maddala, G. S.; Wu, S. 1999. A comparative study of unit root tests with panel data and a new simple test, Oxford Bulletin of Economics and statistics 61 (S1): 631-652.

http://dx.doi.org/10.1111/1468-0084.61.s1.13

Marelli, E.; Signorelli, M. 2010. Employment, productivity and models of growth in the EU, International Journal of Manpower 31(7): 732-754. http://dx.doi.org/10.1108/01437721011081572

Michie, J.; Smith, J. 1997. Employment and economic performance: jobs, inflation, and growth. Oxford University Press, USA. 262 p.

Motley, B. 1998. Growth and inflation: a cross-country study: economic review. Federal Reserve Bank of San Francisco, 15-28.

Nakov, A. A.; Pescatori, A. 2010. Oil and the great moderation, The Economic Journal 120(543): 131-156. http://dx.doi.org/10.1111/j.1468-0297.2009.02302.x

Narayan, P. K.; Narayan, S. 2010. Is there a unit root in the inflation rate? New evidence from panel data models with multiple structural breaks, Applied Economics 42(13): 1661-1670. http://dx.doi.org/10.1080/00036840701721596

Nickell, S. 1998. Unemployment: questions and some answers, The Economic Journal 108(448): 802-816. http://dx.doi.org/10.1111/1468-0297.00316

Nyblom, J.; Harvey, A. 2000. Tests of common stochastic trends, Econometric Theory 16(2): 176-199. http://dx.doi.org/10.1017/S0266466600162024

O'Connell, P. G. J. 1998. The overvaluation of purchasing power parity, Journal of International Economics 44(1): 1-19. http://dx.doi.org/10.1016/S0022-1996(97)00017-2

Okun, A. M. 1980. Postwar macroeconomic performance, in M. Feldstein (Ed.). The American economy in transition. University of Chicago Press, 163-170.

Okun, A. M. 1981. Prices and quantities: a macroeconomic analysis. Brookings Institution. 367 p.

Pedroni, P. 1999. Critical values for cointegration tests in heterogeneous panels with multiple regressors, Oxford Bulletin of Economics and statistics 61(1): 653-670. http://dx.doi.org/10.1111/1468-0084.61.s1.14

Pedroni, P. 2001. Purchasing power parity tests in cointegrated panels, Review of Economics and Statistics 83(4): 727-731. http://dx.doi.org/10.1162/003465301753237803

Pedroni, P. 2004. Panel cointegration: asymptotic and finite sample properties of pooled time series tests with an application to the PPP hypothesis, Econometric Theory 20(03): 597-625.

http://dx.doi.org/10.1017/S0266466604203073 
Persyn, D.; Westerlund, J. 2008. Error-correction-based cointegration tests for panel data, Stata Journal 8(2): 232-241.

Pesaran, M. H. 2007. A simple panel unit root test in the presence of cross section dependence, Journal of Applied Econometrics 22(2): 265-312. http://dx.doi.org/10.1002/jae.951

Pesaran, M. H. 2004. General diagnostic tests for cross section dependence in panels. Cambridge working papers in economics, University of Cambridge, 1-41.

Pesaran, M. H.; Shin, Y.; Smith, R. P. 1999. Pooled mean group estimation of dynamic heterogeneous panels, Journal of the American Statistical Association 94(446): 621-634. http://dx.doi.org/10.1080/01621459.1999.10474156

Pesaran, M. H.; Smith, R. 1995. Estimating long-run relationships from dynamic heterogeneous panels, Journal of Econometrics 68(1): 79-113. http://dx.doi.org/10.1016/0304-4076(94)01644-F

Phelps, E. S. 1967. Phillips curves, expectations of inflation and optimal unemployment over time, Economica 34(135): 254-281. http://dx.doi.org/10.2307/2552025

Phelps, E. S. 1994. Structural slumps: the modern equilibrium theory of unemployment, interest, and assets. Harvard University Press. 420 p.

Phelps, E. S.; Zoega, G. 1998. Natural rate theory and OECD Unemployment, The Economic Journal 108(448): 782-801. http://dx.doi.org/10.1111/1468-0297.00315

Phillips, A. W. 1958. The relation between unemployment and the rate of change of money wage rates in the United Kingdom, 1861-1957, Economica 25(100): 283-299.

Phillips, A. W. 1962. Employment, inflation and growth, Economica 29(113):1-16. http://dx.doi.org/10.1111/j.1468-0335.1962.tb00001.x

Pissarides, C. A.; Vallanti, G. 2004. Productivity growth and employment: theory and panel estimates. CEP Discussion Papers No. dp0663. London School of Economics and Political Science, Centre for Economic Performance. $36 \mathrm{p}$.

Scott, R. H.; McKean, J. 1964. A "cross section" look at employment, growth, and inflation, Economic Inquiry 3(1):1-6. http://dx.doi.org/10.1111/j.1465-7295.1964.tb00899.x

Semko, R. 2013.Optimal economic policy and oil prices shocks in Russia, Ekonomska istrazivanja - Economic Research 26(2): 364-379.

Sill, K. 2011. Inflation dynamics and the new Keynesian Phillips curve, Federal Reserve Bank of Philadelphia Business Review: 1-9.

Šergo, Z.; Saftić, D.; Težak, A. 2012. Stability of Phillips curve: the case of Croatia, Ekonomska istrazivanja Economic Research 25(1): 65-85.

Škare, M. 2010. Can there be a 'golden triangle' of internal equilibrium?, Journal of Policy Modeling 32(4): 562-573. http://dx.doi.org/10.1016/j.jpolmod.2010.05.005

Targetti, F. 1992. Nicholas Kaldor: the economics and politics of capitalism as a dynamic system. USA: Oxford University Press. $416 \mathrm{p}$.

Tatoglu, F. Y. 2011. The long and short run effects between unemployment and economic growth in Europe, Doğuş Üniversitesi Dergisi 12(1): 99-113.

Tobin, J. 1982. Essays in economics: theory and policy. MIT Press. 685 p.

Tobin, J. 1987. Essays in economics: macroeconomics, vol. 1. The MIT Press. 526 p.

Tobin, J. 1997. Full employment and growth: further Keynesian essays on policy. Cheltenham: Edward Elgar Publishing. $312 \mathrm{p}$.

Westerlund, J. 2008. Essays on panel cointegration. Lund Economic Studies No. 129. VDM Verlag. 193 p. 


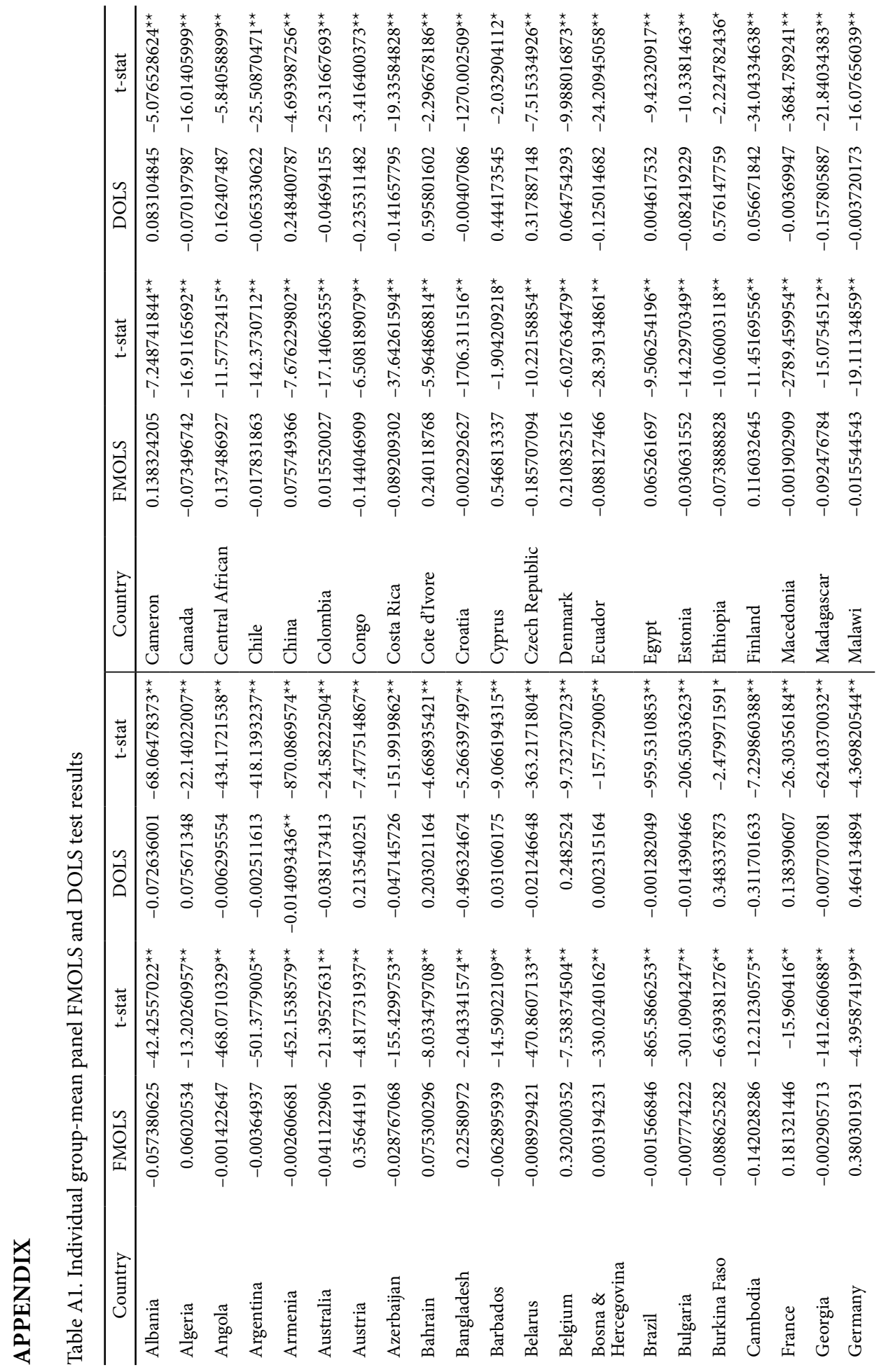




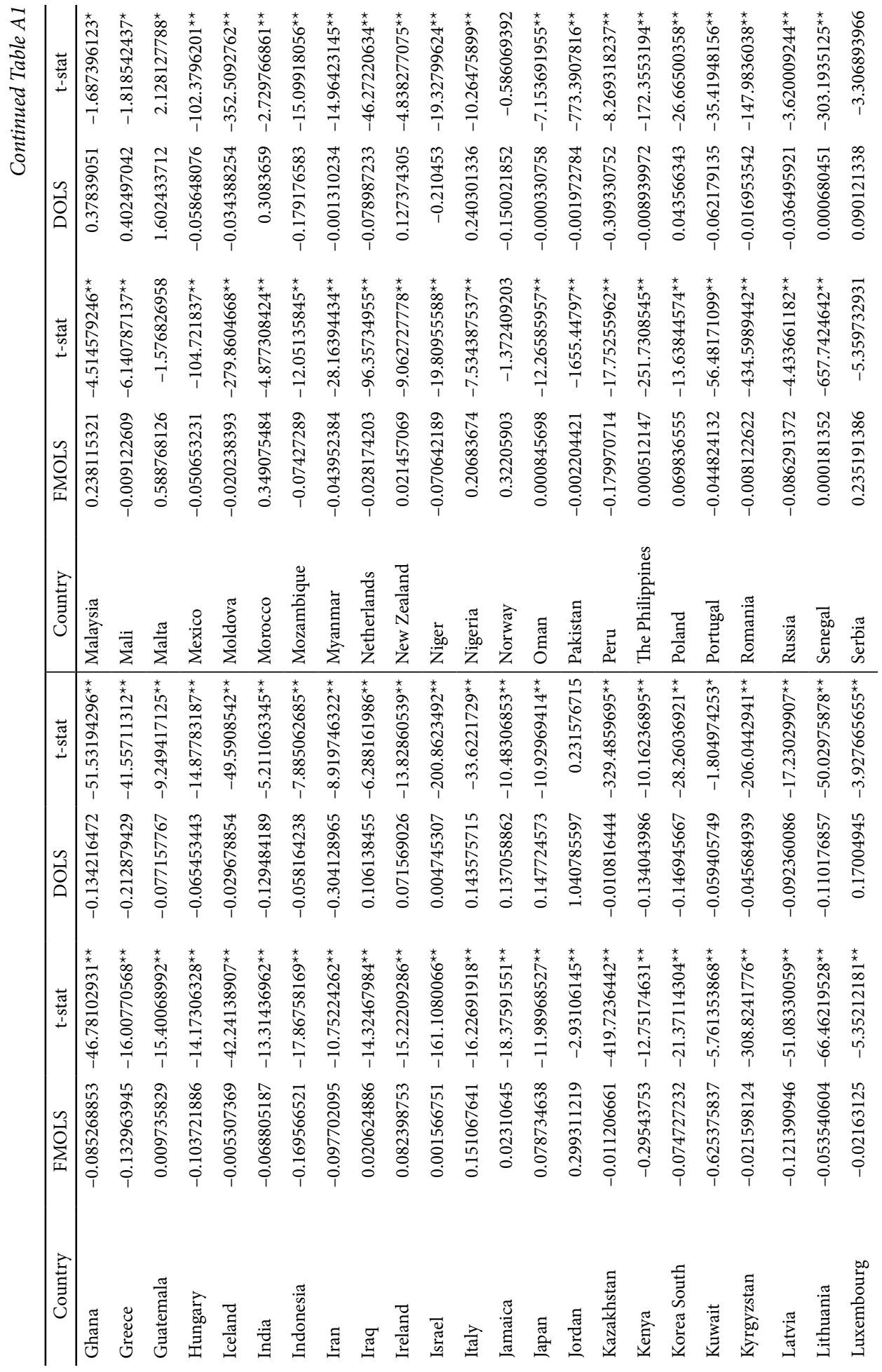




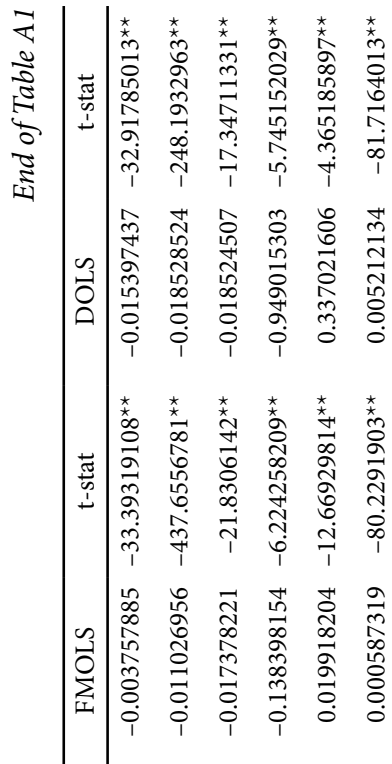

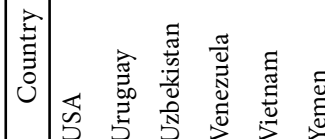

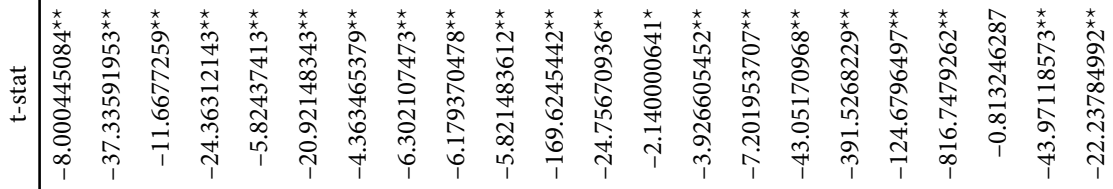

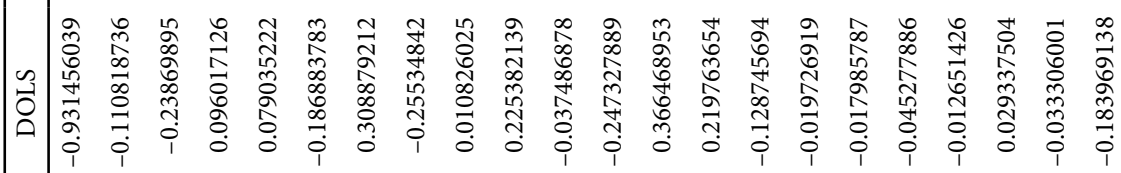

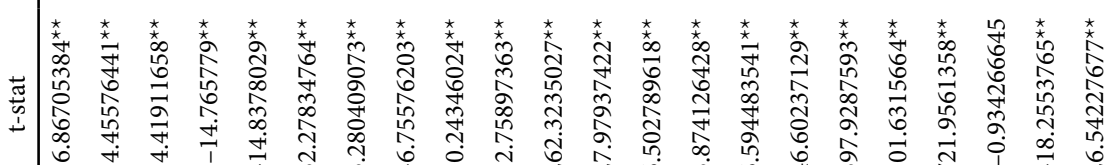

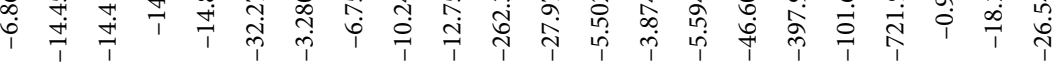

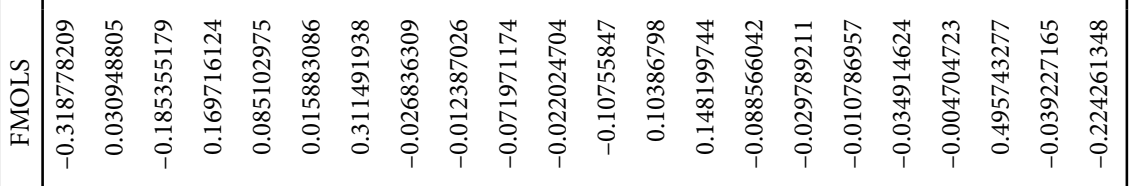

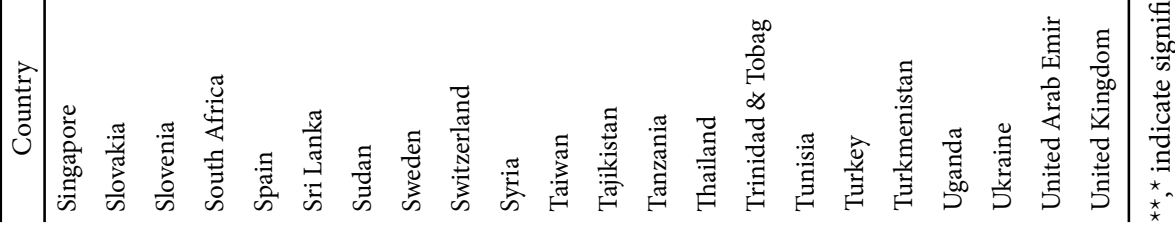




\begin{tabular}{|c|c|c|c|c|c|c|c|c|c|c|c|c|c|c|c|c|c|c|}
\hline ереиеว & $\frac{9}{n}$ & 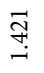 & $\begin{array}{l}8 \\
\stackrel{0}{0} \\
\end{array}$ & $\underset{\Xi}{\beth}$ & $\stackrel{\mathfrak{\sigma}}{\circ}$ & $\begin{array}{l}\text { ro } \\
\stackrel{0}{0}\end{array}$ & ర్రి & ণ্ণ & 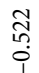 & 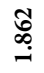 & $\begin{array}{l}\text { กิ } \\
\text { กิ } \\
\text { in }\end{array}$ & 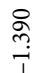 & స్రి & $\stackrel{\text { }}{\leftrightarrows}$ & $\underset{i}{\stackrel{\$}{*}}$ & R̂R & $\begin{array}{l}\hat{0} \\
\stackrel{0}{0}\end{array}$ & 命 \\
\hline นо.әшеว & $\stackrel{\partial}{\circ}$ & તิ & $\stackrel{\infty}{\infty}$ & $\begin{array}{l}\text { ț } \\
\stackrel{+}{0}\end{array}$ & 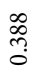 & 웜 & 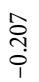 & $\begin{array}{l}\stackrel{\infty}{+} \\
\stackrel{+}{+}\end{array}$ & 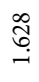 & 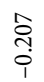 & 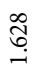 & $\begin{array}{c}\infty \\
\stackrel{+}{+} \\
\stackrel{1}{1}\end{array}$ & $\stackrel{\text { fo }}{\circ}$ & $\stackrel{\vec{\infty}}{\stackrel{m}{0}}$ & ઼ָ & $\stackrel{\vec{n}}{\stackrel{0}{0}}$ & 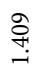 & カั \\
\hline е!poque & $\begin{array}{l}1 \\
\text { ô } \\
0 \\
0\end{array}$ & $\stackrel{\sim}{\stackrel{n}{-}}$ & $\begin{array}{l}\infty \\
\stackrel{\infty}{\infty} \\
\stackrel{0}{0}\end{array}$ & $\begin{array}{l}\stackrel{\infty}{\hat{n}} \\
\sim \\
\sim\end{array}$ & 今̊ & $\hat{\sigma}$ & 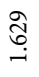 & ๙ू & $\stackrel{\vec{n}}{\stackrel{\sim}{i}}$ & 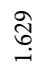 & $\stackrel{\vec{n}}{\stackrel{i}{i}}$ & ฉ̊ & 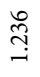 & 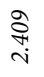 & స్ & $\begin{array}{l}\stackrel{0}{0} \\
\text { in }\end{array}$ & 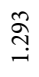 & $\underset{\mathscr{L}}{\stackrel{\mathscr{Z}}{v}}$ \\
\hline it eu!y.nng & \begin{tabular}{l}
\multirow{\sigma}{*}{} \\
$\stackrel{-}{-}$
\end{tabular} & ڤે & 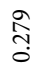 & $\frac{\Re}{\stackrel{2}{0}}$ & ঐু & 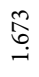 & $\underset{\text { ปิ }}{\stackrel{-}{-}}$ & 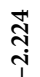 & 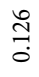 & $\underset{-}{\tilde{G}}$ & $\underset{\stackrel{0}{7}}{\stackrel{0}{0}}$ & 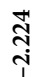 & $\stackrel{\overbrace{}}{\stackrel{\overbrace{}}{i}}$ & $\stackrel{\Re}{\stackrel{9}{*}}$ & 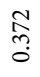 & $\stackrel{\circ}{\stackrel{\infty}{\circ}}$ & $\stackrel{\substack{n \\
0}}{0}$ & $\begin{array}{l}\text { ఫి } \\
\text { i் }\end{array}$ \\
\hline 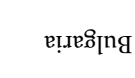 & 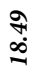 & 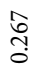 & م̂ & $\frac{\stackrel{9}{6}}{6}$ & $\stackrel{m}{n}$ & 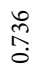 & $\underset{\hat{N}}{\stackrel{N}{\delta}}$ & $\stackrel{\vec{q}}{\stackrel{i}{i}}$ & $\begin{array}{l}\stackrel{\circ}{\infty} \\
\stackrel{1}{i} \\
i\end{array}$ & 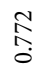 & 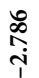 & $\underset{\mathfrak{r}}{i}$ & 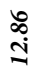 & 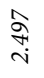 & 京 & 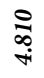 & $\stackrel{\infty}{\stackrel{\infty}{\beth}}$ & $\stackrel{N}{N}$ \\
\hline IIze.xg & $\stackrel{\circ}{\stackrel{\circ}{-}}$ & ণ্ & $\begin{array}{l}\infty \\
\stackrel{0}{0} \\
0 \\
0\end{array}$ & $\stackrel{\widetilde{\sigma}}{0}$ & 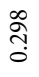 & $\begin{array}{l}\infty \\
\stackrel{0}{0} \\
\stackrel{0}{0}\end{array}$ & $\begin{array}{l}\text { Fै } \\
\text { Oे }\end{array}$ & 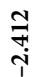 & $\stackrel{\sim}{\stackrel{\overbrace{}}{\leftrightarrows}}$ & 害 & $\underset{\sim}{\stackrel{\overbrace{}}{\rightarrow}}$ & $\underset{i}{\stackrel{7}{7}}$ & $\stackrel{\circ}{\stackrel{0}{n}}$ & 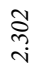 & $\begin{array}{l}\text { 苦 } \\
\text { in }\end{array}$ & 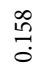 & $\begin{array}{l}\text { @ } \\
\stackrel{1}{7} \\
0\end{array}$ & 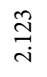 \\
\hline HIG & $\begin{array}{l}\hat{~} \mathscr{0} \\
0\end{array}$ & $\underset{ت}{\stackrel{H}{I}}$ & $\underset{i}{\stackrel{8}{ }}$ & $\stackrel{\vec{n}}{\vec{\sim}}$ & 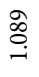 & 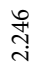 & $\begin{array}{l}\text { fै } \\
\text { กे } \\
\text { i }\end{array}$ & 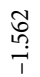 & $\frac{n}{\sigma}$ & $\begin{array}{c}\text { f } \\
\text { m. } \\
\text { i }\end{array}$ & $\frac{10}{\sigma}$ & $\begin{array}{l}\widetilde{\sigma} \\
\stackrel{2}{n} \\
i\end{array}$ & 苞 & 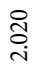 & $\stackrel{10}{\underset{7}{\rightleftarrows}}$ & $\underset{+}{\stackrel{F}{+}}$ & $\begin{array}{l}\infty \\
\infty \\
\infty \\
\infty\end{array}$ & 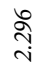 \\
\hline un!̣ి[ə] & 㢳 & $\underset{\sim}{\stackrel{\infty}{\leftrightarrows}}$ & 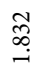 & 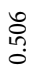 & $\underset{\substack{\infty \\
-\infty}}{\infty}$ & ڤે & $\underset{\tilde{\sigma}}{\tilde{0}}$ & \begin{tabular}{l}
$\infty$ \\
\multirow{0}{0}{} \\
$i$ \\
$i$
\end{tabular} & 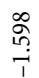 & $\underset{\widehat{C}}{0}$ & 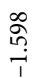 & 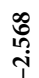 & $\begin{array}{l}\frac{10}{0} \\
\stackrel{0}{0}\end{array}$ & 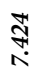 & $\begin{array}{l}\vec{n} \\
\stackrel{\infty}{-}\end{array}$ & $\underset{\hat{\sigma}}{\sigma}$ & $\stackrel{m}{n}$ & $\stackrel{\infty}{\stackrel{\Upsilon}{i}}$ \\
\hline sn.xp[əg & $\begin{array}{l}\text { जे } \\
\text { के }\end{array}$ & $\stackrel{n}{n}$ & $\begin{array}{l}\hat{k} \\
\hat{\sigma}\end{array}$ & 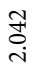 & 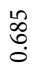 & $\underset{\sigma}{\sigma}$ & గ్రి & 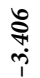 & \begin{tabular}{l}
$\infty$ \\
\multirow{1}{n}{} \\
0 \\
1
\end{tabular} & 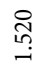 & 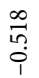 & 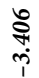 & $\begin{array}{l}\hat{B} \\
+ \\
+\end{array}$ & ڤે & $\underset{6}{0}$ & $\begin{array}{l}\text { H' } \\
\text { in } \\
\dot{n}\end{array}$ & $\begin{array}{l}\text { तु } \\
\stackrel{0}{0} \\
0\end{array}$ & $\underset{\text { in }}{\widehat{N}}$ \\
\hline sopequeg & $\stackrel{\infty}{\infty}$ & $\underset{\infty}{\stackrel{n}{0}}$ & $\stackrel{\widehat{\sigma}}{\text { - }}$ & O্ণ & $\underset{b}{\stackrel{7}{\sharp}}$ & 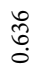 & 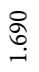 & 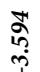 & $\stackrel{n}{\tilde{N}}$ & 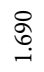 & 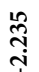 & $\begin{array}{l}\text { मे } \\
\text { ñ } \\
\text { n. }\end{array}$ & ô & $\frac{\infty}{0}$ & 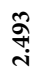 & $\stackrel{\infty}{\stackrel{\circ}{\circ}}$ & $\underset{\text { กี }}{\overrightarrow{+}}$ & $\underset{\text { in }}{\stackrel{t}{0}}$ \\
\hline чsәре [̊̊ueg & ڤे & $\stackrel{\widetilde{ల}}{0}$ & $\stackrel{\infty}{\underset{7}{*}}$ & 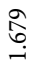 & $\underset{\infty}{\mathscr{\infty}}$ & $\underset{j}{\sharp}$ & \begin{tabular}{l}
\multirow{H}{*}{} \\
$\stackrel{1}{0}$
\end{tabular} & 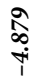 & $\underset{\overbrace{}}{\stackrel{\Upsilon}{0}}$ & $\begin{array}{l}\text { Z } \\
\text { ô } \\
\text { i }\end{array}$ & 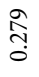 & 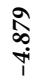 & $\stackrel{n}{\tilde{n}} \underset{0}{0}$ & $\begin{array}{l}\ddot{0} \\
: \\
\stackrel{0}{0}\end{array}$ & $\begin{array}{l}\infty \\
\stackrel{+}{+} \\
\dot{m}\end{array}$ & $\underset{i}{\stackrel{9}{i}}$ & $\underset{\stackrel{3}{0}}{\stackrel{n}{0}}$ & ळे \\
\hline u!̣exueg & 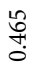 & 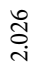 & 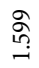 & $\frac{\circ}{6}$ & $\begin{array}{l}\vec{n} \\
\stackrel{0}{0} \\
\stackrel{0}{0}\end{array}$ & 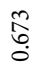 & 융 & گ̆ & 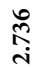 & $\begin{array}{l}\text { 융 } \\
\text { 잉 }\end{array}$ & 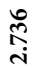 & $\underset{-}{\stackrel{-}{-}}$ & 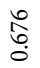 & $\begin{array}{l}\infty \\
\stackrel{\infty}{\rightarrow}\end{array}$ & $\stackrel{n}{7}$ & $\underset{\sim}{\stackrel{\infty}{二}}$ & $\begin{array}{l}\hat{L} \\
\hat{b} \\
\stackrel{i}{1}\end{array}$ & $\begin{array}{l}\infty \\
\stackrel{\infty}{0} \\
0\end{array}$ \\
\hline ue!̣̣eq.ızZ & $\underset{\vec{i}}{\overrightarrow{\mathrm{d}}}$ & $\begin{array}{l}\stackrel{\circ}{\circ} \\
\text { ᄋ }\end{array}$ & $\underset{\text { i }}{\stackrel{\infty}{8}}$ & Б્ડ & $\begin{array}{l}\text { त్ర } \\
0 \\
0\end{array}$ & $\stackrel{\widehat{?}}{\stackrel{?}{0}}$ & $\underset{\text { 오 }}{\text { N }}$ & 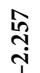 & $\begin{array}{l}\text { ⿵人丶 } \\
\text { ô }\end{array}$ & 命 & $\begin{array}{l}\text { ले } \\
\text { ñ } \\
0\end{array}$ & กิ & $\begin{array}{l}\infty \\
\stackrel{\infty}{q} \\
\stackrel{4}{v}\end{array}$ & ลે & $\underset{\sim}{\mathbb{R}}$ & $\begin{array}{l}\widetilde{\widehat{C}} \\
\text { i }\end{array}$ & $\begin{array}{l}\text { in } \\
\text { ț } \\
0\end{array}$ & $\stackrel{\infty}{\stackrel{్}{్}}$ \\
\hline ห!̣ısก & $\begin{array}{l}\hat{\&} \\
\stackrel{0}{0}\end{array}$ & م̂n & $\begin{array}{l}n \\
\infty \\
\infty \\
0 \\
0\end{array}$ & $\begin{array}{l}18 \\
80 \\
10\end{array}$ & 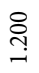 & $\stackrel{\text { ஸे }}{\text { ஸे }}$ & $\stackrel{\stackrel{2}{\leftrightarrows}}{\underset{0}{0}}$ & $\stackrel{\mathrm{a}}{\mathrm{i}}$ & $\begin{array}{l}1 \\
\stackrel{0}{0} \\
0\end{array}$ & $\stackrel{\stackrel{2}{7}}{\underset{0}{0}}$ & $\begin{array}{l}\text { S. } \\
\infty \\
0\end{array}$ & 옹 & 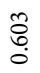 & $\begin{array}{l}\text { ले } \\
\text { ڤ్ర }\end{array}$ & $\stackrel{\text { స్ }}{\text { సે }}$ & $\begin{array}{l}\circ \\
\dot{\gamma}\end{array}$ & $\begin{array}{l}8 \\
\stackrel{0}{0} \\
0\end{array}$ & $\underset{\dot{m}}{\stackrel{\Xi}{ٍ}}$ \\
\hline eโןex\}sn & 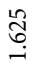 & స̂. & $\stackrel{\vec{\delta}}{0}$ & 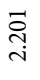 & $\stackrel{\infty}{\underset{N}{N}}$ & 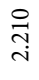 & $\underset{\text { ㅇ }}{\stackrel{\text { i }}{ }}$ & $\begin{array}{l}\hat{L} \\
\qquad 0 \\
i \\
i\end{array}$ & 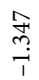 & $\begin{array}{l}\text { Oे } \\
\text { i }\end{array}$ & $\stackrel{\text { 年 }}{\rightarrow}$ & $\begin{array}{l}10 \\
b \\
i \\
i\end{array}$ & 命 & 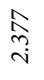 & \begin{tabular}{l}
+ \\
\multirow{0}{0}{} \\
0 \\
0
\end{tabular} & 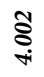 & $\underset{\text { in }}{\stackrel{7}{7}}$ & $\underset{\sim}{\mathbb{f}}$ \\
\hline 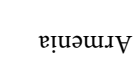 & $\frac{1}{8}$ & $\stackrel{\overrightarrow{0}}{\overrightarrow{0}}$ & $\begin{array}{l}\text { مू } \\
\text { ó }\end{array}$ & ণ্రి & $\stackrel{\text { İ }}{\circ}$ & $\begin{array}{l}\infty \\
\stackrel{0}{=} \\
=\end{array}$ & $\begin{array}{l}\overrightarrow{0} \\
\text { nె? }\end{array}$ & 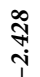 & $\stackrel{n}{n} \hat{n}$ & $\begin{array}{l}\overrightarrow{0} \\
\text { ֻn } \\
0\end{array}$ & $\stackrel{n}{\tilde{n}}$ & $\underset{\sim}{\stackrel{\infty}{*}} \underset{\sim}{\sim}$ & $\begin{array}{l}\text { ș } \\
\text { వి }\end{array}$ & 今ે & 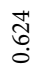 & 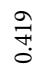 & 울 & $\begin{array}{l}\vec{N} \\
\dot{N}\end{array}$ \\
\hline 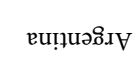 & $\begin{array}{l}\infty \\
\stackrel{0}{\infty} \\
0 \\
0\end{array}$ & 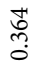 & $\stackrel{\infty}{\rightrightarrows}$ & ণั) & $\begin{array}{l}1 \\
0 \\
0\end{array}$ & $\stackrel{\infty}{=}$ & $\begin{array}{l}\infty \\
\hat{\tilde{\sigma}} \\
0\end{array}$ & $\begin{array}{l}\stackrel{\text { సे }}{\infty} \\
\stackrel{+}{i}\end{array}$ & 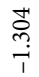 & \begin{tabular}{l}
$\infty$ \\
\multirow{ల}{0}{} \\
0
\end{tabular} & ণ্ণি & 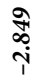 & 怘 & $\frac{\infty}{h}$ & $\begin{array}{l}\text { \& } \\
\text { in }\end{array}$ & $\begin{array}{l}\text { బి } \\
\text { N̦ }\end{array}$ & $\underset{0}{\circ}$ & $\underset{\substack{\infty \\
i}}{\stackrel{\infty}{i}}$ \\
\hline 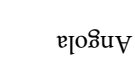 & $\underset{\mathrm{\sigma}}{\stackrel{N}{*}}$ & 총 & $\begin{array}{l}\text { rot } \\
\stackrel{0}{0}\end{array}$ & ণু & $\stackrel{n}{\tilde{n}}$ & 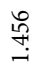 & \begin{tabular}{l}
\multirow{2}{0}{} \\
$\stackrel{n}{1}$ \\
$i$
\end{tabular} & $\begin{array}{l}\overrightarrow{\widetilde{C}} \\
\stackrel{i}{i}\end{array}$ & $\begin{array}{l}\stackrel{\hat{\vartheta}}{6} \\
0\end{array}$ & \begin{tabular}{l}
5 \\
\multirow{5}{*}{} \\
$i$
\end{tabular} & 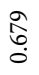 & $\begin{array}{l}\vec{B} \\
i \\
i\end{array}$ & $\underset{\tilde{N}}{\tilde{n}}$ & $\underset{i}{\stackrel{\infty}{i}}$ & $\begin{array}{l}\text { గ్ } \\
\stackrel{0}{0} \\
0\end{array}$ & $\stackrel{\vec{n}}{\stackrel{v}{v}}$ & ㅎํ & $\stackrel{m}{\stackrel{m}{v}}$ \\
\hline 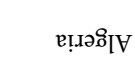 & 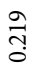 & $\begin{array}{l}\text { in } \\
\text { ì }\end{array}$ & $\begin{array}{l}\text { t' } \\
\stackrel{n}{0} \\
0\end{array}$ & $\begin{array}{l}\stackrel{\infty}{\infty} \\
\underset{\sim}{N}\end{array}$ & 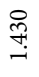 & 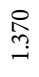 & $\begin{array}{l}\not \\
\infty \\
0 \\
0 \\
i\end{array}$ & ণั & $\stackrel{\text { ஸે }}{\text { ஸे }}$ & $\begin{array}{l}+ \\
\mathbb{0} \\
0 \\
0 \\
0 \\
1\end{array}$ & ஸ્ & 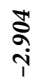 & $\begin{array}{l}n \\
\text { ஸn } \\
\sigma\end{array}$ & $\underset{\sim}{\stackrel{\sim}{\sim}}$ & 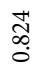 & 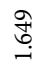 & $\stackrel{\infty}{\stackrel{\infty}{\sigma}}$ & $\begin{array}{c}\tilde{m} \\
\tilde{n}\end{array}$ \\
\hline e!̣ueqIV & $\underset{\widetilde{\infty}}{\stackrel{\infty}{二}}$ & ণ্ণী & $\frac{2}{1+}$ & ণ্ণ & $\underset{\widetilde{\infty}}{\stackrel{0}{0}}$ & $\stackrel{m}{\stackrel{0}{0}}$ & $\begin{array}{l}\stackrel{0}{0} \\
\stackrel{0}{0}\end{array}$ & $\underset{\sim}{\stackrel{7}{\sim}}$ & $\begin{array}{l}\text { ̊̊ } \\
\text { ¿ }\end{array}$ & 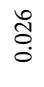 & 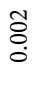 & 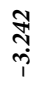 & $\widehat{\varnothing}$ & ڤ్రి & $\underset{\stackrel{3}{+}}{\stackrel{0}{0}}$ & $\underset{0}{\stackrel{N}{S}}$ & $\underset{\substack{0 \\
0}}{0}$ & $\begin{array}{l}\vec{H} \\
\stackrel{0}{0} \\
\dot{r}\end{array}$ \\
\hline & $\stackrel{i}{i}$ & $\stackrel{\lambda}{\hat{\imath}}$ & $\stackrel{i}{\hat{\imath}}$ & $\stackrel{\imath}{\uparrow}$ & $\stackrel{i}{i}$ & डे & $\stackrel{i}{i}$ & $\vec{i}$ & $\dot{\imath}$ & $\stackrel{\uparrow}{\uparrow}$ & $\stackrel{i}{i}$ & $\widehat{\uparrow}$ & $\stackrel{Q}{1}$ & $\stackrel{\lambda}{i}$ & $\dot{\uparrow}$ & $\stackrel{\imath}{\uparrow}$ & $\stackrel{\ddot{\uparrow}}{\lambda}$ & $\vec{\uparrow}$ \\
\hline & & & 采 & & & & & & - & & & & & & & & & \\
\hline
\end{tabular}




\begin{tabular}{|c|c|c|c|c|c|c|c|c|c|c|c|c|c|c|c|c|c|c|}
\hline витчЭ & $\begin{array}{l}\text { ळे } \\
\text { के }\end{array}$ & 总 & ন & 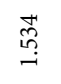 & 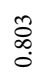 & $\underset{\oplus}{\stackrel{m}{\oplus}}$ & $\begin{array}{l}\text { 웅 } \\
\text { ind } \\
i\end{array}$ & $\begin{array}{l}\tilde{n} \\
\hat{0} \\
i \\
i\end{array}$ & $\underset{\overbrace{}}{\overparen{్}}$ & 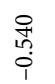 & $\underset{\overbrace{}}{\mathscr{乛}}$ & $\begin{array}{l}n \\
\tilde{S} \\
i \\
i\end{array}$ & $\mid \begin{array}{l}\overline{9} \\
\text { के }\end{array}$ & 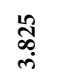 & $\begin{array}{l}\stackrel{\infty}{\sim} \\
\sim\end{array}$ & $\underset{\widetilde{\Xi}}{\widetilde{i}}$ & 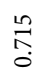 & $\begin{array}{l}\vec{F} \\
\stackrel{0}{i} \\
i\end{array}$ \\
\hline 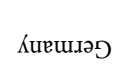 & $\underset{\text { సี }}{\stackrel{\sim}{n}}$ & 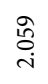 & $\stackrel{\infty}{\stackrel{\infty}{\rightarrow}}$ & $\stackrel{\text { 蒀 }}{-}$ & $\underset{\overbrace{}}{\stackrel{n}{+}}$ & $\stackrel{\stackrel{\leftrightarrow}{\mathrm{i}}}{\mathrm{i}}$ & $\stackrel{\overrightarrow{3}}{\stackrel{7}{+}}$ & $\begin{array}{l}\underset{7}{7} \\
\underset{i}{i}\end{array}$ & 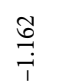 & $\stackrel{\vec{f}}{\rightarrow}$ & 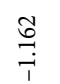 & $\begin{array}{l}\underset{7}{7} \\
\underset{i}{i}\end{array}$ & $\underset{⿱}{\stackrel{\vec{T}}{\mathbf{T}}}$ & 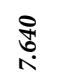 & 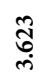 & 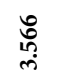 & $\stackrel{م}{\stackrel{N}{i}}$ & 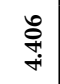 \\
\hline 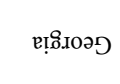 & $\underset{\text { i }}{\vec{i}}$ & $\stackrel{\infty}{\stackrel{一}{\leftrightarrow}}$ & $\bar{\sigma}$ & $\begin{array}{l}\text { ț } \\
\text { aे }\end{array}$ & 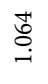 & $\begin{array}{l}\text { d̆ } \\
\text { ì }\end{array}$ & $\underset{m}{m}$ & 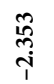 & $\underset{+}{\stackrel{+}{0}}$ & 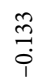 & 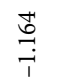 & 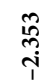 & $\stackrel{\overrightarrow{0}}{\rightarrow}$ & $\begin{array}{l}0 \\
0 \\
10 \\
10\end{array}$ & $\stackrel{\infty}{\stackrel{\infty}{-}}$ & बे & $\underset{\overbrace{}}{\stackrel{\overbrace{}}{\hookrightarrow}}$ & $\begin{array}{l}\text { बे } \\
\text { iे }\end{array}$ \\
\hline әәие. स $_{\mathrm{A}}$ & 缹 & $\underset{\widetilde{\sigma}}{\tilde{r}}$ & 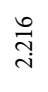 & $\begin{array}{l}0 \\
\stackrel{0}{0}\end{array}$ & ڤે. & 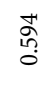 & $\stackrel{\stackrel{n}{?}}{\rightarrow}$ & 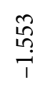 & $\underset{i}{\stackrel{N}{T}}$ & $\stackrel{\stackrel{n}{f}}{\rightarrow}$ & $\underset{\sim}{\stackrel{N}{T}}$ & $\stackrel{\substack{\hat{n} \\
\rightarrow}}{\rightarrow}$ & $\begin{array}{l}\widetilde{N} \\
\infty \\
0 \\
0\end{array}$ & $\begin{array}{l}\stackrel{n}{R} \\
\text { in }\end{array}$ & 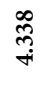 & $\begin{array}{l}\stackrel{\triangleright}{\circ} \\
\stackrel{0}{0}\end{array}$ & $\begin{array}{l}\stackrel{\mathbb{N}}{\mathrm{N}} \\
\text { o }\end{array}$ & 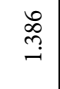 \\
\hline pue[u!t] & $\begin{array}{l}\hat{\text { ते }} \\
\text { i் }\end{array}$ & $\underset{\hat{i}}{\stackrel{2}{ה}}$ & ف영 & 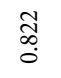 & 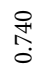 & तु. & 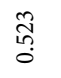 & 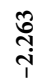 & ণิ ָ̊ & ్ָָ̃ & ڤ̊ํํ & $\underset{\substack{\mathrm{C} \\
i}}{i}$ & $\overrightarrow{\hat{\sigma}}$ & $\hat{\mathscr{o}}$ & $\begin{array}{l}\stackrel{n}{h} \\
0 \\
0\end{array}$ & $\begin{array}{l}\stackrel{2}{2} \\
\stackrel{0}{0}\end{array}$ & 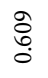 & 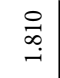 \\
\hline 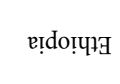 & ڤે & ণั ָे & $\overrightarrow{0}$ & $\begin{array}{l}\widetilde{S} \\
\infty \\
0\end{array}$ & ఫ్ర్రి & $\frac{\overrightarrow{0}}{0}$ & $\stackrel{\circ}{\stackrel{\infty}{\rightarrow}}$ & ふે & $\begin{array}{l}\stackrel{\infty}{\circ} \\
\stackrel{0}{0}\end{array}$ & $\stackrel{\infty}{\stackrel{\infty}{-}}$ & $\begin{array}{l}\stackrel{\infty}{\infty} \\
\stackrel{0}{0}\end{array}$ & & $\begin{array}{l}\infty \\
\infty \\
\infty \\
+ \\
+\end{array}$ & $\underset{+}{\stackrel{0}{7}}$ & $\begin{array}{l}n \\
: 0 \\
0 \\
0\end{array}$ & 怘 & $\begin{array}{l}\stackrel{0}{\infty} \\
\text { o. }\end{array}$ & 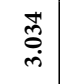 \\
\hline ห̣̣uołs & $\stackrel{\sqrt[5]{3}}{=}$ & $\begin{array}{l}\overrightarrow{\widetilde{N}} \\
\stackrel{0}{0}\end{array}$ & $\begin{array}{l}\stackrel{\hat{0}}{\infty} \\
\text { i }\end{array}$ & $\begin{array}{l}\stackrel{D}{\check{D}} \\
\dot{m}\end{array}$ & $\underset{\infty}{\stackrel{\infty}{9}}$ & भ̆ & 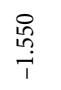 & $\underset{i}{\stackrel{\mathcal{T}}{i}}$ & $\begin{array}{l}\text { H } \\
\stackrel{H}{+} \\
\stackrel{i}{i}\end{array}$ & $\underset{\substack{0 \\
\hdashline}}{\stackrel{8}{1}}$ & $\begin{array}{l}\vec{H} \\
\stackrel{+}{+} \\
\stackrel{+}{i}\end{array}$ & $\underset{i}{\mathcal{F}}$ & 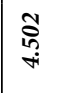 & ลू & 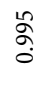 & $\underset{\infty}{\infty}$ & $\begin{array}{l}\tilde{8} \\
\text { in } \\
\end{array}$ & $\approx$ \\
\hline $\mathrm{l} \mathrm{d} / \mathrm{K}_{\mathrm{B}}$ & $\begin{array}{l}\infty \\
\stackrel{\infty}{0} \\
0 \\
0\end{array}$ & $\begin{array}{l}\infty \\
\stackrel{\infty}{\circ} \\
\stackrel{0}{0}\end{array}$ & $\stackrel{\circ}{\circ}$ & 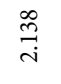 & 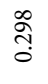 & $\begin{array}{l}\stackrel{0}{0} \\
0 \\
0\end{array}$ & $\begin{array}{l}\text { ते } \\
\stackrel{\circ}{0} \\
\end{array}$ & $\begin{array}{l}\infty \\
\stackrel{0}{p} \\
i\end{array}$ & 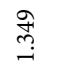 & $\begin{array}{l}\text { 䓪 } \\
\stackrel{0}{0}\end{array}$ & $\stackrel{\text { fे }}{\stackrel{9}{-}}$ & $\begin{array}{l}m \\
\tilde{p} \\
\dot{p}\end{array}$ & ले & $\stackrel{\tilde{a}}{\overrightarrow{+}}$ & $\begin{array}{l}\hat{0} \\
\stackrel{0}{0}\end{array}$ & 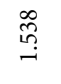 & $\begin{array}{l}\infty \\
\infty \\
\infty \\
0 \\
0\end{array}$ & 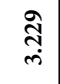 \\
\hline Iopenot & $\begin{array}{l}\text { : } \\
\stackrel{0}{0}\end{array}$ & đ̊ & 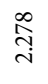 & $\stackrel{\vec{F}}{\mathrm{i}}$ & 犬્స & $\frac{\Re}{\mathbb{O}}$ & $\begin{array}{l}\vec{n} \\
\hat{n} \\
0\end{array}$ & $\stackrel{\widehat{N}}{\hat{i}}$ & 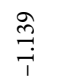 & $\begin{array}{l}\vec{n} \\
\text { ñ } \\
\text {. }\end{array}$ & 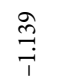 & $\underset{\hat{i}}{\hat{T}}$ & $\frac{\text { I }}{0}$ & 苫 & 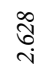 & 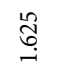 & 苮 & 员 \\
\hline ұльешиә & 今ે & 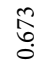 & $\stackrel{m}{m}$ & 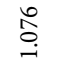 & 众 & 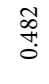 & $\stackrel{\infty}{\stackrel{0}{0}}$ & $\underset{7}{\stackrel{7}{p}}$ & $\begin{array}{l}\text { مू } \\
\stackrel{+}{i}\end{array}$ & $\stackrel{\infty}{\stackrel{\infty}{0}}$ & $\begin{array}{l}\text { no } \\
\stackrel{0}{i}\end{array}$ & 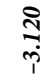 & $\stackrel{2}{a}$ & 芯 & $\underset{i n}{\stackrel{9}{\rightarrow}}$ & $\begin{array}{l}\text { S } \\
\stackrel{0}{0}\end{array}$ & స్స్ & $\underset{i n}{7}$ \\
\hline ·dəપ્વ ЧગәzЈ & $\begin{array}{l}\widetilde{0} \\
\stackrel{0}{0}\end{array}$ & $\begin{array}{l}\vec{J} \\
\text { is }\end{array}$ & $\stackrel{\stackrel{9}{\stackrel{9}{ت}}}{-}$ & $\begin{array}{l}\vec{\Delta} \\
\stackrel{0}{0} \\
0\end{array}$ & $\begin{array}{l}\text { ĥ } \\
\text { î }\end{array}$ & $\begin{array}{l}\stackrel{\Re}{\varrho} \\
\stackrel{2}{2}\end{array}$ & $\begin{array}{l}\text { : } \\
\stackrel{0}{0} \\
0\end{array}$ & 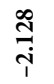 & $\begin{array}{l}\text { 年 } \\
6 \\
i \\
i\end{array}$ & $\begin{array}{l}\stackrel{8}{0} \\
\stackrel{0}{0}\end{array}$ & 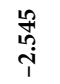 & $\underset{i}{\stackrel{\infty}{T}}$ & 合 & $\begin{array}{l}\stackrel{H}{n} \\
\text { in } \\
\text { in }\end{array}$ & \begin{tabular}{l}
$\tilde{D}$ \\
\multirow{0}{0}{} \\
$\dot{r}$
\end{tabular} & $\begin{array}{l}\vec{J} \\
\infty \\
0\end{array}$ & $\begin{array}{l}\stackrel{\infty}{\infty} \\
\stackrel{+}{+}\end{array}$ & ஸ̂ \\
\hline sn.diאכ & $\begin{array}{l}\text { Fేّ } \\
\text { Oे }\end{array}$ & $\stackrel{n}{m}$ & $\stackrel{0}{=}$ & $\stackrel{+}{\stackrel{0}{0}}$ & $\begin{array}{l}\stackrel{0}{\circ} \\
\stackrel{0}{0} \\
0\end{array}$ & ते & $\hat{\alpha}$ & 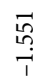 & 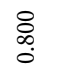 & $\hat{\sigma}$ & 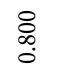 & $\begin{array}{l}\vec{n} \\
\stackrel{n}{n} \\
i\end{array}$ & $\begin{array}{l}\text { 告 } \\
\text { f̊ } \\
0\end{array}$ & 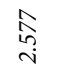 & 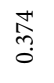 & 梁 & 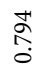 & ف․ \\
\hline שו̣poxว & 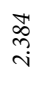 & 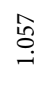 & §ิ & $\begin{array}{l}8 \\
\text { के }\end{array}$ & $\begin{array}{l}\text { స' } \\
\text { aे }\end{array}$ & $\underset{-}{\vec{\infty}}$ & $\stackrel{\text { స్ }}{\text { ৷ }}$ & $\begin{array}{l}\stackrel{3}{7} \\
\underset{1}{7}\end{array}$ & $\underset{\mathfrak{T}}{\mathbb{T}}$ & ন্ & $\underset{\mathfrak{T}}{\mathbb{T}}$ & $\begin{array}{l}\stackrel{8}{7} \\
\ddot{1}\end{array}$ & 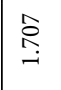 & $\begin{array}{l}\hat{⿵} \\
\hat{h} \\
r \\
r\end{array}$ & 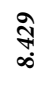 & 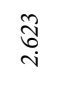 & $\begin{array}{l}\text { 今े } \\
\text { గn }\end{array}$ & $\underset{\text { స్ర }}{\overrightarrow{+}}$ \\
\hline 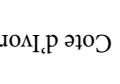 & 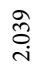 & 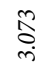 & ڤે̀ & $\begin{array}{l}\text { ్ָరి } \\
\stackrel{\sim}{i}\end{array}$ & $\underset{\text { i }}{\stackrel{\text { i }}{i}}$ & $\stackrel{\text { 㟔 }}{\rightarrow}$ & $\begin{array}{c}\hat{\sigma} \\
\hat{i}\end{array}$ & $\begin{array}{l}\text { ڤั) } \\
\text { ஸे }\end{array}$ & $\frac{m}{m}$ & ஸे & $\frac{m}{m}$ & ڤి & $\underset{+}{\pi}$ & $\underset{\substack{\hat{\infty} \\
\text { i }}}{ }$ & $\stackrel{B}{=}$ & 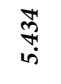 & 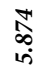 & 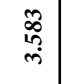 \\
\hline 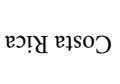 & శ్రి & శ్రి & ใ్ & $\begin{array}{l}\infty \\
\stackrel{\infty}{0} \\
\dot{n}\end{array}$ & 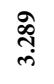 & $\underset{\vec{n}}{\vec{i}}$ & 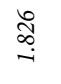 & $\begin{array}{l}\underset{0}{0} \\
\stackrel{i}{i}\end{array}$ & 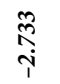 & 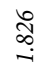 & 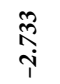 & $\begin{array}{l}\text { ते } \\
\stackrel{i}{i}\end{array}$ & बे & $\underset{\stackrel{\rho}{\infty}}{\stackrel{\rho}{\dot{m}}}$ & $\stackrel{n}{n}$ & 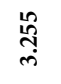 & 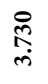 & $\hat{\tilde{~}}$ \\
\hline oถ̊นoว & $\stackrel{\overbrace{}}{\mathscr{\theta}}$ & $\underset{\overparen{J}}{\stackrel{H}{0}}$ & $\stackrel{\leftrightarrow}{\stackrel{2}{0}}$ & $\underset{\text { \&̊ }}{\infty}$ & ถู & $\begin{array}{l}\hat{\sigma} \\
\stackrel{0}{0}\end{array}$ & $\stackrel{\infty}{\stackrel{\infty}{-}}$ & $\begin{array}{l}\hat{2} \\
\hat{i} \\
\hat{i}\end{array}$ & $\begin{array}{l}\stackrel{\infty}{0} \\
\text { i }\end{array}$ & 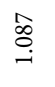 & $\begin{array}{l}\infty \\
\stackrel{\leftrightarrow}{0}\end{array}$ & $\begin{array}{l}\hat{2} \\
\hat{i} \\
\hat{i}\end{array}$ & $\stackrel{\text { సิ }}{-}$ & $\begin{array}{l}\hat{N} \\
\hat{0}\end{array}$ & $\stackrel{\mathscr{I}}{\stackrel{1}{-}}$ & $\begin{array}{l}\stackrel{0}{0} \\
0 \\
0\end{array}$ & $\underset{\mathrm{i}}{\stackrel{\text { f }}{\text { in }}}$ & $\underset{f}{\stackrel{n}{7}}$ \\
\hline в!quоo & $\stackrel{\Re}{=}$ & $\stackrel{\infty}{\stackrel{\infty}{0}}$ & $\begin{array}{l}\stackrel{o}{\circ} \\
\stackrel{0}{0}\end{array}$ & ๙ૂ & F⿱艹⿹勹巳 & $\begin{array}{l}\infty \\
\stackrel{0}{0} \\
0\end{array}$ & $\begin{array}{l}\text { त్ } \\
\text { r }\end{array}$ & $\begin{array}{l}\underset{\infty}{\Delta} \\
\stackrel{i}{i}\end{array}$ & $\begin{array}{c}\text { ले } \\
\hat{n} \\
i \\
i\end{array}$ & श్రి & $\begin{array}{l}\text { ले } \\
\text { in } \\
\text { i }\end{array}$ & $\begin{array}{l}\underset{D}{\infty} \\
i \\
i\end{array}$ & $\begin{array}{l}6 \\
\text { in } \\
\text { in }\end{array}$ & 蒿 & $\stackrel{\sharp}{\stackrel{3}{0}}$ & $\begin{array}{l}\ddot{l} \\
\infty \\
+\end{array}$ & $\begin{array}{l}\text { Oे } \\
\text { I! } \\
0\end{array}$ & 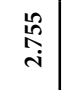 \\
\hline หuापว & よे & 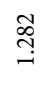 & $\stackrel{\sim}{\underset{N}{\sim}}$ & ণু & 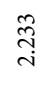 & $\underset{\square}{ت}$ & $\underset{i}{\stackrel{3}{T}}$ & 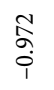 & $\underset{\substack{n \\
i}}{\stackrel{\infty}{i}}$ & 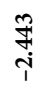 & 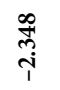 & $\begin{array}{l}\text { N } \\
\text { Q }\end{array}$ & 文 & 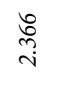 & 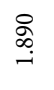 & $\begin{array}{l}\tilde{O} \\
\text { ì }\end{array}$ & 今 & 总 \\
\hline ગ!ણ૫ & 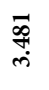 & $\underset{6}{8}$ & $\stackrel{\pi}{m}$ & $\stackrel{\mathscr{\Omega}}{\tilde{O}}$ & ふֶ. & ஓ̊. & $\underset{\mathrm{o}}{\stackrel{0}{\circ}}$ & $\begin{array}{l}\underset{\alpha}{ } \\
\stackrel{i}{i}\end{array}$ & $\begin{array}{l}\stackrel{8}{0} \\
\stackrel{0}{i}\end{array}$ & $\stackrel{\leftrightarrow}{\stackrel{8}{\circ}}$ & $\begin{array}{l}\otimes \\
\stackrel{0}{0} \\
i\end{array}$ & 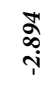 & 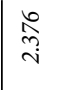 & 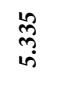 & $\begin{array}{l}\infty \\
\stackrel{8}{0} \\
\stackrel{0}{0}\end{array}$ & $\underset{0}{\vec{F}}$ & $\underset{\mathrm{\sigma}}{\overrightarrow{\mathrm{C}}}$ & $\stackrel{\approx}{\circ}$ \\
\hline 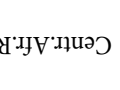 & : & 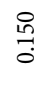 & $\vec{\sigma}$ & I̊ & $\underset{\mathrm{N}}{\overrightarrow{\mathrm{N}}}$ & $\begin{array}{l}\infty \\
\stackrel{0}{0} \\
0\end{array}$ & 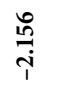 & 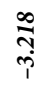 & $\underset{\rightarrow}{\vec{T}}$ & $\underset{i}{\stackrel{n}{i}}$ & $\underset{\hat{T}}{\hat{T}}$ & $\begin{array}{l}\stackrel{\infty}{\overrightarrow{7}} \\
\underset{i}{i}\end{array}$ & $\frac{\tilde{c}}{\dot{n}}$ & $\underset{m}{\stackrel{m}{r}}$ & 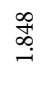 & 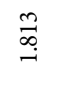 & 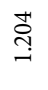 & $\begin{array}{c}\hat{n} \\
\hat{n} \\
\dot{m}\end{array}$ \\
\hline & $\begin{array}{l}\hat{\uparrow} \\
\lambda\end{array}$ & $\bar{\uparrow}$ & $\begin{array}{l}\tilde{\uparrow} \\
\end{array}$ & $\stackrel{\uparrow}{\uparrow}$ & $\begin{array}{l}i \\
\lambda\end{array}$ & & $\begin{array}{l}i \\
\lambda \\
\lambda\end{array}$ & $\begin{array}{l}\lambda \\
\hat{\imath} \\
\end{array}$ & $\begin{array}{l}i \\
i \\
2\end{array}$ & i & $\begin{array}{l}i \\
i\end{array}$ & $\uparrow$ & $\begin{array}{l}\uparrow \\
\uparrow \\
\lambda\end{array}$ & $\begin{array}{l}\lambda \\
i \\
2\end{array}$ & $\approx$ & $\stackrel{\uparrow}{\uparrow}$ & 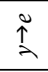 & $\lambda$ \\
\hline & & & జี & & & & & & 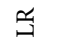 & & & & & & & & & \\
\hline
\end{tabular}




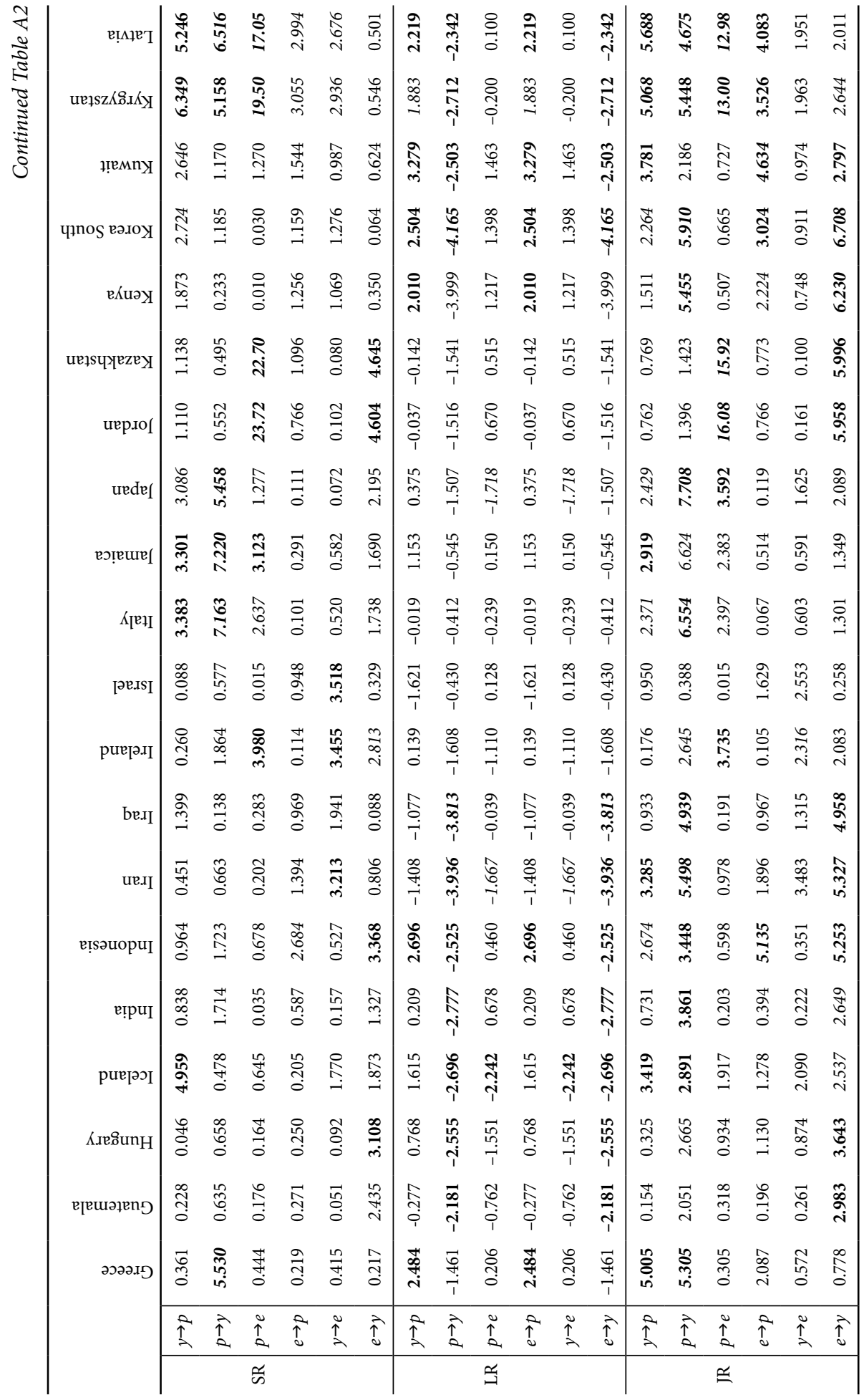




\begin{tabular}{|c|c|c|c|c|c|c|c|c|c|c|c|c|c|c|c|c|c|c|}
\hline 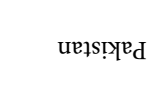 & $\stackrel{\infty}{\stackrel{\infty}{\sigma}}$ & $\stackrel{\Re}{\stackrel{C}{I}}$ & ڤิ & 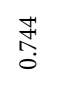 & $\begin{array}{l}\text { H } \\
\text { ñ } \\
0\end{array}$ & 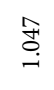 & İ & $\underset{i}{\mathbb{T}}$ & $\begin{array}{l}\infty \\
\infty \\
h \\
i \\
i \\
i\end{array}$ & $\underset{\Xi}{\overparen{O}}$ & $\begin{array}{l}\infty \\
\infty \\
\text { in } \\
\text { in } \\
1\end{array}$ & 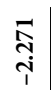 & 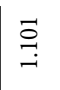 & 离 & $\stackrel{\text { กิ }}{\text { กิ }}$ & ‡ั & 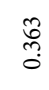 & 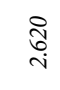 \\
\hline uвшо & ત̂న & $\stackrel{\sim}{\stackrel{\sim}{+}}$ & $\begin{array}{l}\text { ro } \\
\text { in } \\
0\end{array}$ & $\begin{array}{l}\infty \\
\stackrel{\infty}{0} \\
\stackrel{0}{0}\end{array}$ & $\begin{array}{l}\overrightarrow{\vec{t}} \\
\vec{i}\end{array}$ & 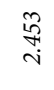 & 年 & $\underset{i}{\stackrel{n}{h}}$ & $\underset{i}{\stackrel{\oplus}{i}}$ & 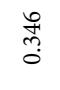 & $\underset{i}{\stackrel{\oplus}{i}}$ & $\underset{i}{\stackrel{h}{\stackrel{h}{2}}}$ & 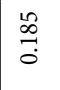 & ஸి & $\underset{\stackrel{\circ}{\circ}}{\infty}$ & $\stackrel{0}{\stackrel{0}{0}}$ & 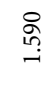 & ત્ર \\
\hline KeM.ION & $\stackrel{7}{\sharp}$ & 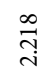 & 융 & $\stackrel{0}{0}$ & $\stackrel{\widehat{\aleph}}{i}$ & ลे & $\overrightarrow{\stackrel{\rho}{\circ}}$ & $\underset{\text { Sे }}{\text { gे }}$ & ণิ ָָ & $\stackrel{\vec{\rho}}{\dot{\rho}}$ & ণิ ָָ & $\underset{\mathrm{i}}{\mathrm{i}}$ & $\begin{array}{l}\text { H. } \\
0 \\
0 \\
0\end{array}$ & $\stackrel{\Re}{\check{C}}$ & $\stackrel{\infty}{\stackrel{I}{I}}$ & $\begin{array}{l}\stackrel{0}{0} \\
\stackrel{8}{0} \\
0\end{array}$ & 芯 & ఫั \\
\hline หฺ̣วร์!N & 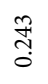 & $\begin{array}{l}\stackrel{\infty}{\infty} \\
\overbrace{0}^{0}\end{array}$ & 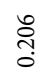 & 范 & $\vec{\delta}$ & 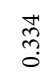 & $\begin{array}{l}\stackrel{a}{0} \\
\stackrel{0}{0} \\
\dot{1}\end{array}$ & $\begin{array}{l}\vec{n} \\
\underset{1}{1} \\
\text { In }\end{array}$ & $\underset{-\infty}{\infty}$ & $\begin{array}{l}\stackrel{a}{\infty} \\
\stackrel{0}{1}\end{array}$ & $\underset{\substack{\infty \\
-i}}{\stackrel{m}{\infty}}$ & $\begin{array}{l}\overrightarrow{\mathfrak{n}} \\
\underset{i}{i}\end{array}$ & 今ે & 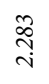 & 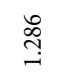 & $\begin{array}{l}\infty \\
\stackrel{\infty}{0}\end{array}$ & $\stackrel{m}{\stackrel{3}{*}}$ & 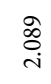 \\
\hline เวอ!ก & $\underset{\widetilde{\pi}}{\widetilde{0}}$ & 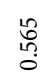 & 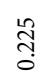 & $\begin{array}{l}\infty \\
\stackrel{\infty}{n} \\
0\end{array}$ & $\begin{array}{l}+ \\
\stackrel{5}{0} \\
0\end{array}$ & $\overrightarrow{\widetilde{r}}$ & $\begin{array}{l}\infty \\
\stackrel{\infty}{0} \\
0 \\
1\end{array}$ & $\begin{array}{l}\infty \\
\stackrel{\infty}{i} \\
\stackrel{i}{i}\end{array}$ & $\stackrel{\substack{0 \\
o}}{o}$ & $\begin{array}{l}\stackrel{\infty}{1} \\
\stackrel{1}{1}\end{array}$ & 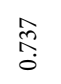 & $\begin{array}{l}\stackrel{\mathscr{O}}{\mathrm{o}} \\
\stackrel{i}{i}\end{array}$ & ڤั & $\underset{\dot{m}}{\bar{j}}$ & $\begin{array}{l}\stackrel{n}{n} \\
\tilde{n} \\
0\end{array}$ & $\stackrel{\substack{n \\
0}}{\stackrel{0}{0}}$ & $\stackrel{\vec{\partial}}{\stackrel{-}{0}}$ & $\begin{array}{l}\text { స్ } \\
\text { లి }\end{array}$ \\
\hline рuг[еәZ мәN & 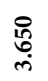 & $\stackrel{\widetilde{N}}{i}$ & $\stackrel{\infty}{\stackrel{\infty}{\rightarrow-}}$ & है & 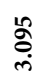 & 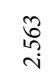 & 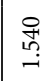 & $\frac{\mathbb{T}}{+i}$ & 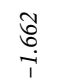 & $\stackrel{\text { 윰 }}{\stackrel{\leftrightarrow}{n}}$ & $\underset{\stackrel{0}{0}}{\stackrel{0}{-}}$ & $\underset{\mathbb{T}}{\stackrel{7}{+}}$ & స్ & $\stackrel{\mathbb{T}}{\stackrel{i}{Z}}$ & 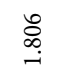 & 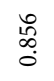 & $\begin{array}{l}\text { ปั } \\
\text { స్ }\end{array}$ & $\stackrel{\infty}{\infty}$ \\
\hline sрuеןдәчдәN & స్రీ & $\stackrel{\infty}{\stackrel{\circ}{\circ}}$ & $\stackrel{\stackrel{\leftrightarrow}{\circ}}{\stackrel{-}{-}}$ & $\begin{array}{l}\infty \\
: \\
\dot{m}\end{array}$ & $\underset{\overbrace{}}{\stackrel{\overbrace{}}{\leftrightarrows}}$ & $\begin{array}{l}\text { స్రે } \\
\text { ì }\end{array}$ & 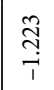 & $\stackrel{8}{\stackrel{\circ}{h}}$ & $\underset{\stackrel{\infty}{T}}{\stackrel{\infty}{i}}$ & 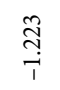 & $\underset{\stackrel{\infty}{T}}{\stackrel{\infty}{i}}$ & $\underset{\substack{0 \\
\hdashline}}{\stackrel{8}{1}}$ & 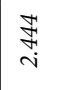 & ڤે & $\begin{array}{l}\stackrel{0}{0} \\
\infty \\
0\end{array}$ & ठे & $\stackrel{\mathscr{\infty}}{=}$ & 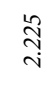 \\
\hline seuuue $_{\mathrm{W}}$ & $\underset{+}{\stackrel{5}{+}}$ & ڤे & $\vec{\Delta}$ & $\begin{array}{l}\vec{\sigma} \\
\dot{0}\end{array}$ & बे & 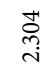 & 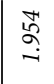 & 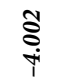 & $\underset{i}{\stackrel{尺}{i}}$ & $\stackrel{\text { 岕 }}{-}$ & $\underset{i}{\stackrel{\hat{i}}{i}}$ & $\underset{\Im}{\tilde{+}}$ & ర్d & $\stackrel{\widehat{̣}}{=}$ & $\stackrel{\widetilde{\Omega}}{\stackrel{-}{\Omega}}$ & $\underset{+}{\vec{F}}$ & 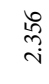 & స్ \\
\hline 'quirzow & ஜ̂ & 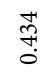 & $\vec{b}$ & 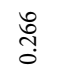 & $\stackrel{\infty}{=}$ & $\begin{array}{l}\text { f } \\
\text { 号 }\end{array}$ & $\underset{\substack{\tilde{i} \\
i}}{i}$ & 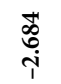 & 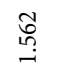 & 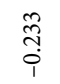 & مْ & $\begin{array}{l}\overrightarrow{0} \\
\stackrel{0}{0} \\
i\end{array}$ & $\vec{b}$ & $\begin{array}{l}\stackrel{0}{\circ} \\
\text { i }\end{array}$ & $\begin{array}{l}\text { 足 } \\
\text { o. } \\
0\end{array}$ & 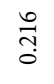 & 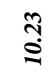 & ఫ̊ \\
\hline оговон & $\underset{\stackrel{H}{*}}{\stackrel{H}{\circ}}$ & 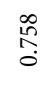 & $\stackrel{\circ}{\stackrel{0}{0}}$ & 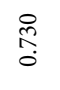 & 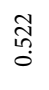 & تٕ: & $\stackrel{\infty}{\stackrel{2}{7}}$ & $\underset{i}{\stackrel{i}{i}}$ & $\underset{i}{\mathbb{i}}$ & $\stackrel{\infty}{\stackrel{\infty}{\leftrightarrows}}$ & $\underset{i}{\mathrm{i}}$ & $\underset{i}{\stackrel{i}{i}}$ & ్ֶt & $\begin{array}{l}\text { O̊. } \\
\text { in }\end{array}$ & 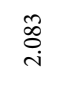 & 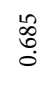 & $\stackrel{\sqrt[n]{3}}{\stackrel{7}{3}}$ & $\underset{\mathrm{T}}{\mathrm{T}}$ \\
\hline вморгон & $\underset{\overparen{H}}{\stackrel{\varpi}{+}}$ & $\stackrel{\infty}{\vec{n}}$ & $\stackrel{?}{1}$ & $\stackrel{\substack{n\\
}}{-}$ & $\begin{array}{l}\mathscr{0} \\
\stackrel{్}{్}\end{array}$ & $\begin{array}{l}\stackrel{\circ}{\circ} \\
\stackrel{\leftrightarrow}{\circ}\end{array}$ & 会 & $\underset{\substack{\hat{n} \\
\text { in } \\
i}}{1}$ & స్ & $\stackrel{\hat{A}}{\stackrel{\hat{i}}{ }}$ & స్ & 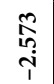 & $\vec{r}$ & $\stackrel{m}{\vec{j}}$ & $\stackrel{\infty}{\infty}$ & $\begin{array}{l}\stackrel{W}{N} \\
\text { i }\end{array}$ & $\stackrel{\mathscr{g}}{\mathrm{g}}$ & 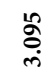 \\
\hline оэ!्रәण & 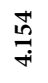 & \begin{tabular}{l}
$\infty$ \\
\multirow{2}{0}{} \\
0
\end{tabular} & $\stackrel{0}{\substack{0 \\
0}}$ & $\begin{array}{l}\infty \\
\stackrel{0}{\circ}\end{array}$ & $\begin{array}{l}\stackrel{2}{6} \\
\stackrel{\sim}{i}\end{array}$ & $\begin{array}{l}\hat{\sigma} \\
\text { o }\end{array}$ & $\underset{i}{\stackrel{0}{c}}$ & 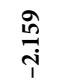 & $\underset{\mathbb{T}}{\mathbb{i}}$ & $\underset{\text { i }}{\stackrel{0}{0}}$ & $\underset{\mathbb{T}}{\tilde{i}}$ & $\stackrel{\substack{10 \\
i}}{i}$ & ò & 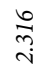 & $\underset{\stackrel{L}{q}}{\stackrel{\sim}{\sim}}$ & 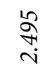 & ণ্ & ָे \\
\hline eqfe्w & 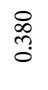 & ఫี & 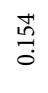 & $\begin{array}{l}0 \\
0 \\
0\end{array}$ & \begin{tabular}{l}
$\stackrel{\circ}{n}$ \\
\multirow{2}{0}{}
\end{tabular} & $\stackrel{\infty}{\stackrel{\infty}{\rightarrow}}$ & $\begin{array}{l}\infty \\
\stackrel{2}{\hat{~}} \\
\hat{i}\end{array}$ & 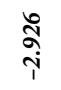 & $\underset{i}{i}$ & $\begin{array}{l}\stackrel{\infty}{\hat{N}} \\
\hat{i} \\
\hat{1}\end{array}$ & $\underset{i}{\stackrel{i}{i}}$ & 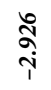 & 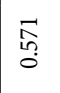 & స్ & ָे & $\begin{array}{l}\text { तิ } \\
\text { กิ }\end{array}$ & $\begin{array}{l}\hat{\sigma} \\
\dot{r}\end{array}$ & $\stackrel{\hat{\sigma}}{\dot{r}}$ \\
\hline 茛 & $\begin{array}{l}\stackrel{0}{0} \\
0 \\
0\end{array}$ & ఫે & 疋 & $\begin{array}{l}\text { त् } \\
\text { ¿ू. }\end{array}$ & $\underset{\substack{n \\
6}}{-}$ & $\begin{array}{l}\infty \\
\stackrel{\infty}{0} \\
i\end{array}$ & 年 & 产 & $\frac{\pi}{n}$ & 今̂े & $\frac{\pi}{\pi}$ & 啇 & ồ & 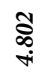 & $\underset{+}{\stackrel{n}{+}}$ & 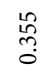 & $\underset{\text { in }}{\mathbb{7}}$ & $\underset{+}{\tilde{\infty}}$ \\
\hline 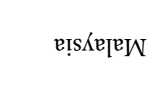 & 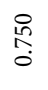 & $\begin{array}{c}\text { ఫ్ } \\
\text { Li }\end{array}$ & $\begin{array}{l}\text { D̊ } \\
\text { గn } \\
0\end{array}$ & $\stackrel{\infty}{\infty}$ & $\stackrel{\stackrel{\sim}{N}}{0}$ & $\begin{array}{l}\stackrel{0}{0} \\
\text { i }\end{array}$ & $\begin{array}{l}\text { Oे } \\
\hat{i} \\
i\end{array}$ & $\stackrel{\tilde{e}}{i}$ & $\frac{2}{9}$ & $\begin{array}{l}\text { کิ } \\
\text { ì }\end{array}$ & $\underset{\hat{i}}{2}$ & $\stackrel{\leftrightarrow}{\stackrel{C}{i}}$ & $\begin{array}{c}\hat{\infty} \\
\stackrel{m}{i n}\end{array}$ & ণ্ণ & $\stackrel{\substack{\hat{m} \\
0}}{0}$ & $\frac{m}{m}$ & $\stackrel{\vec{\infty}}{\stackrel{1}{0}}$ & $\begin{array}{l}\stackrel{゚}{N} \\
\text { N }\end{array}$ \\
\hline IMETEN & 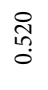 & $\begin{array}{l}\stackrel{\infty}{0} \\
\stackrel{\infty}{\circ}\end{array}$ & 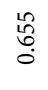 & 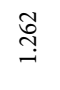 & $\frac{m}{n}$ & $\underset{\stackrel{s}{+}}{\vec{f}}$ & 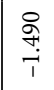 & $\underset{\hat{P}}{\hat{P}}$ & 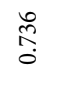 & 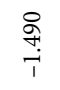 & 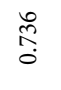 & $\underset{\hat{i}}{\stackrel{N}{i}}$ & 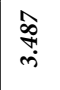 & ڤ్ & $\begin{array}{l}\vec{n} \\
\stackrel{n}{0}\end{array}$ & $\underset{-}{\stackrel{R}{0}}$ & ก̂ & $\begin{array}{l}80 \\
80\end{array}$ \\
\hline 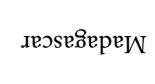 & 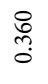 & 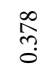 & 劧 & 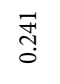 & $\begin{array}{l}\stackrel{0}{0} \\
\infty \\
\infty\end{array}$ & $\stackrel{\text { Sf }}{\rightarrow}$ & $\stackrel{9}{\stackrel{9}{=}}$ & $\underset{7}{\stackrel{7}{i}}$ & $\begin{array}{l}n \\
6 \\
0 \\
\dot{m}\end{array}$ & $\stackrel{\text { m}}{\rightrightarrows}$ & $\begin{array}{l}n \\
\hat{6} \\
\dot{m}\end{array}$ & $\underset{+i}{\stackrel{7}{7}}$ & 告 & $\underset{\text { in }}{\mathbb{7}}$ & 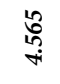 & ڤ్రి & $\stackrel{\infty}{\infty}$ & $\frac{\infty}{6}$ \\
\hline е!̣uорәэећ & : & $\begin{array}{l}\stackrel{0}{\infty} \\
\dot{\oplus}\end{array}$ & $\underset{d}{\stackrel{H}{0}}$ & $\begin{array}{l}\vec{n} \\
\stackrel{0}{0}\end{array}$ & 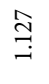 & 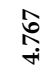 & $\stackrel{\widehat{0}}{=}$ & $\underset{\hat{\Upsilon}}{\hat{i}}$ & 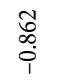 & $\stackrel{\widehat{\sigma}}{\leftrightarrows}$ & $\begin{array}{l}\widetilde{S} \\
\infty \\
0 \\
i\end{array}$ & 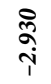 & $\begin{array}{l}\mathfrak{2} \\
0 \\
0\end{array}$ & $\begin{array}{l}\text { 궁 } \\
\text { in }\end{array}$ & वे & $\stackrel{H}{-}$ & 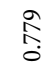 & $\begin{array}{l}\text { క్ } \\
\text { in }\end{array}$ \\
\hline qüxnт & $\underset{ \pm}{ \pm}$ & $\begin{array}{l}\infty \\
\stackrel{\infty}{+} \\
\dot{m}\end{array}$ & $\stackrel{\pi}{2}$ & ల్ల & ঙ્亍 & \begin{tabular}{l}
+ \\
$\stackrel{0}{0}$ \\
\multirow{0}{0}{}
\end{tabular} & 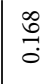 & $\underset{i}{\mathbb{i}}$ & 尔 & $\stackrel{\infty}{\stackrel{\infty}{0}}$ & 丽 & $\underset{i}{\stackrel{H}{i}}$ & 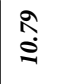 & $\begin{array}{l}\stackrel{\circ}{\circ} \\
\text { i }\end{array}$ & $\underset{\alpha}{k}$ & 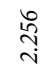 & ఫี & 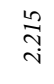 \\
\hline \multirow[t]{3}{*}{ в!̣uenyџ!T } & $\begin{array}{l}\hat{s} \\
\text { is }\end{array}$ & 会 & $\underset{\stackrel{9}{+}}{\stackrel{乛}{+}}$ & $\begin{array}{l}\vec{D} \\
\stackrel{\infty}{\sim}\end{array}$ & $\underset{f}{\stackrel{f}{7}}$ & $\begin{array}{l}\infty \\
\stackrel{0}{0}+1 \\
\vdots\end{array}$ & శ్రి & 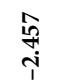 & $\begin{array}{l}\text { o } \\
\text { + } \\
i \\
i\end{array}$ & $\begin{array}{l}\widetilde{0} \\
0 \\
0\end{array}$ & $\begin{array}{l}\text { o } \\
\text { + } \\
\text { i }\end{array}$ & $\underset{\substack{f \\
i \\
i}}{i}$ & $\begin{array}{c}\infty \\
\stackrel{0}{=}\end{array}$ & 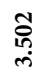 & ๙̊̊ & तે & 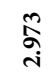 & 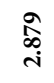 \\
\hline & $\begin{array}{l}2 \\
1 \\
\lambda\end{array}$ & $\vec{i}$ & $\begin{array}{l} \\
i \\
i \\
2\end{array}$ & $\hat{i}_{0}^{2}$ & $\begin{array}{l}i \\
\uparrow \\
\grave{\lambda}\end{array}$ & $\widehat{\imath}$ & $\stackrel{i}{i}$ & $\begin{array}{l}\overrightarrow{\hat{\imath}} \\
\underline{2}\end{array}$ & $\begin{array}{l}\dot{\uparrow} \\
i \\
2\end{array}$ & $\begin{array}{l}\text { i } \\
\hat{\imath}\end{array}$ & $\stackrel{i}{\uparrow}$ & $\overrightarrow{\hat{i}}$ & $\begin{array}{l}2 \\
1 \\
\lambda\end{array}$ & $\begin{array}{l}\vec{i} \\
2\end{array}$ & $\begin{array}{l}0 . \\
\uparrow \\
2\end{array}$ & $\stackrel{i}{i}$ & 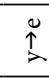 & $\widehat{\imath}$ \\
\hline & & & $\mathscr{E}$ & & & & & & $\stackrel{9}{\exists}$ & & & & & & , & & & \\
\hline
\end{tabular}




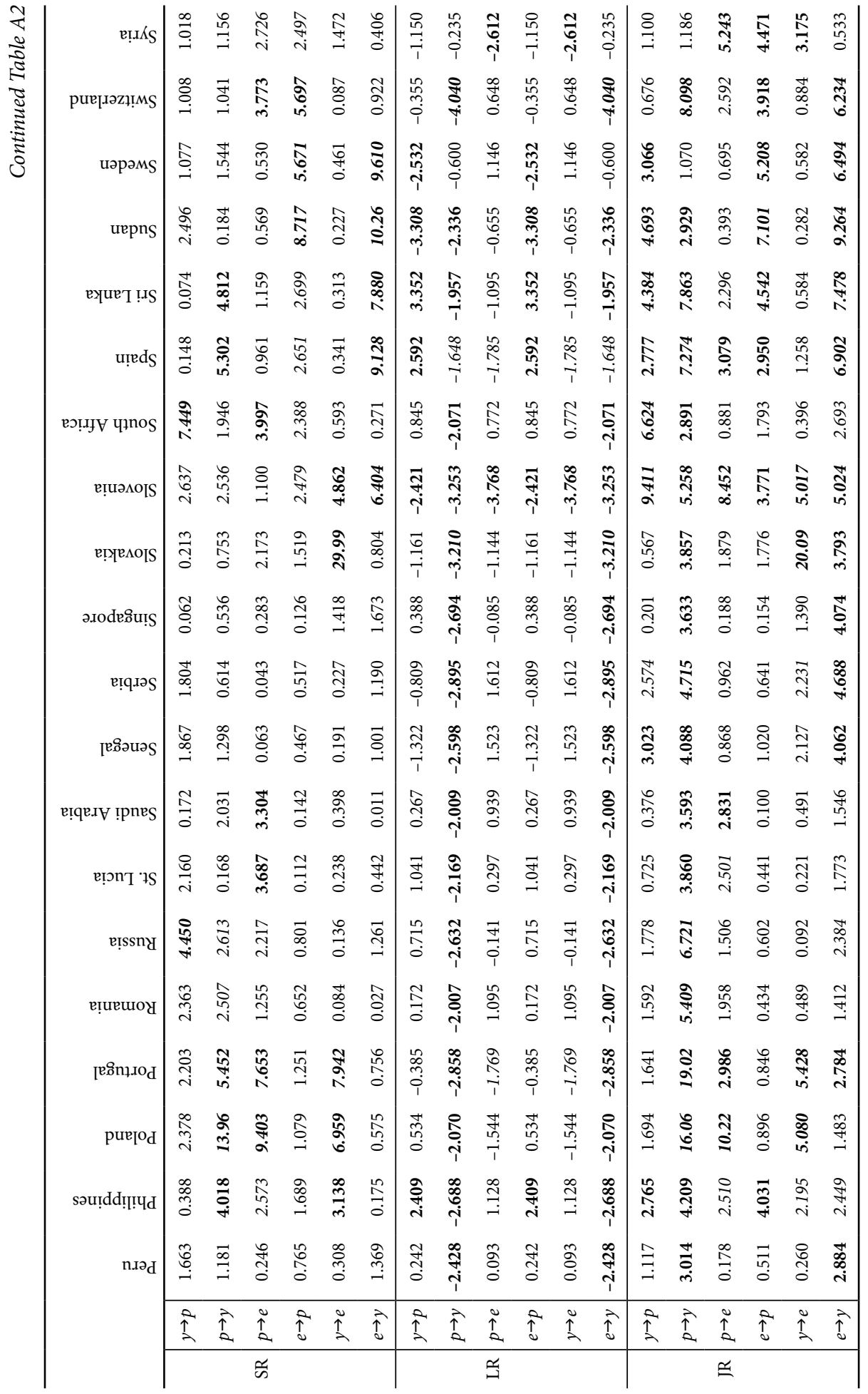




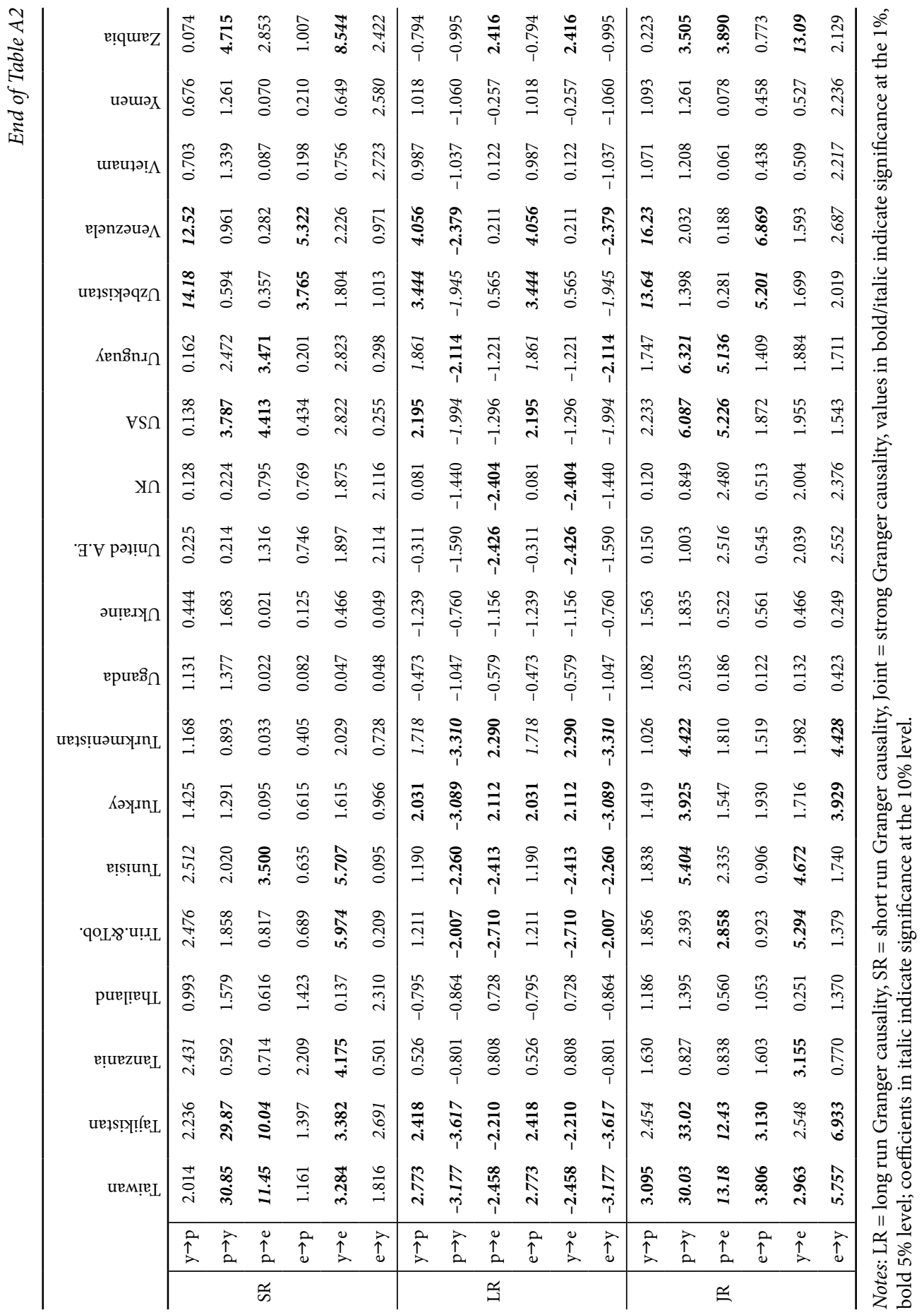


Marinko ŠKARE. Professor of Economics, Economic Research Journal Editor in Chief, Member of Editorial Board of several international journals, Department Economics and Tourism "Dr. Mijo Mirković" in Pula, Juraj Dobrila University of Pula. He served as Assistant Dean for Education, Faculty of Economics \&Tourism, Pula, Assistant Dean for International Cooperation, Faculty of Economics \& Tourism, Pula, Main and Team Researcher on several scientific projects, Former Dean of the Faculty of Economics \& Tourism, Pula and Former Vice President for International Cooperation, Juraj Dobrila University of Pula. He has published several books and a large number of scientific papers on the subject of economic growth, welfare economics and poverty, human capital, economics in transition, economic philosophy and monetary economics. He is a member of the American Economic Association, Royal Economic Society, Economic History Association, Economic History Society, and Association for Comparative Economic Studies.

Guglielmo Maria CAPORALE is Professor of Economics and Finance and Director of the Centre for Empirical Finance at Brunel University, London. He is also a Visiting Professor at London South Bank University and London Metropolitan University, a Research Professor at DIW Berlin, a CESifo Research Network Fellow and an NCID (Navarra Center for International Development) Non-Resident Fellow. Prior to taking up his current position, he was a Research Officer at the National Institute of Economic and Social Research in London; a Research Fellow and then a Senior Research Fellow at the Centre for Economic Forecasting at the London Business School; Professor of Economics at the University of East London; Professor of Economics and Finance as well as Director of the Centre for Monetary and Financial Economics at London South Bank University. 